\title{
Effects of oscillating spacetime metric background on a complex scalar field and formation of topological vortices
}

\author{
Shreyansh S. Dave $\circledast^{1, *}$ and Sanatan Digal ${ }^{1,2, \dagger}$ \\ ${ }^{1}$ The Institute of Mathematical Sciences, Chennai 600113, India \\ ${ }^{2}$ Homi Bhabha National Institute, Training School Complex, Anushakti Nagar, Mumbai 400085, India
}

(Received 12 June 2020; revised 12 March 2021; accepted 6 May 2021; published 8 June 2021)

\begin{abstract}
We study the time evolution of a complex scalar field in the symmetry broken phase in the presence of oscillating spacetime metric background. In our $(2+1)$-dimensional simulations, we show that the spacetime oscillations can excite an initial field configuration, which ultimately leads to the formation of topological vortices in the system. At late times, field configuration achieves a disordered state. A detailed study of the momentum and frequency modes of the field reveals that these field excitations are driven by the phenomenon of parametric resonance. In the extremely-high-frequency regime where frequency of spacetime oscillations is much larger than the field-mass, the formed vortices are not topological in nature. Interestingly, in this regime, for a suitable choice of parameters of the simulation, we observe a persistent lattice structure of vortex-antivortex pairs. We discuss applications of our study to the dynamics of interior superfluidity of neutron stars during binary neutron star mergers, in generation of excitation in ultralight axionlike field near a strong gravitational wave source, etc.
\end{abstract}

DOI: 10.1103/PhysRevD.103.116007

\section{INTRODUCTION}

Topological defects exist in systems ranging from condensed matter to the early Universe [1,2]. They exist in systems which have topologically nontrivial order parameter space (or vacuum manifold) [3]. There are many condensed matter systems, e.g., superfluid, superconductor, nematic liquid crystal, etc., which have topologically nontrivial order parameter space. For example, the superfluid phase of ${ }^{4} \mathrm{He}$ has circle $S^{1}$ as an order parameter space, for which the fundamental group is nontrivial, i.e., $\pi_{1}\left(S^{1}\right)=\mathcal{Z}$ [3]. Therefore, in this system, topological vortices could exist. Topological vortices are characterized by different elements of the fundamental group.

There are various ways by which topological defects can be formed in a physical system. These defects can form during spontaneous symmetry breaking phase transition, the formation of which is described by the Kibble-Zurek mechanism [1,4]. However, in the presence of external influence, this mechanism needs some modifications $[5,6]$. There are other methods also by which these defects can be formed. For example, in superfluid ${ }^{4} \mathrm{He}$, the rotation of a

\footnotetext{
*shreyanshsd@imsc.res.in

†igal@imsc.res.in
}

Published by the American Physical Society under the terms of the Creative Commons Attribution 4.0 International license. Further distribution of this work must maintain attribution to the author(s) and the published article's title, journal citation, and DOI. Funded by SCOAP ${ }^{3}$. vessel within a range of angular velocity leads to the formation of vortex lattice [7,8]. Similarly, in a type-II superconductor, an external magnetic field within a range of field strength leads to the formation of flux-tube lattice $[7,8]$.

In Refs. [9,10], an interesting possibility of production of topological defects is investigated in $\Phi^{4}$ theories. In these studies, defect-antidefect pairs have been produced under oscillating temperature of the effective potential with frequency close to mass of the field. This leads to the parametric resonance of the field, under which the field achieves excitations leading to the formation of vortexantivortex pairs in U(1)-theory [9] and kink-antikink pairs in $\mathcal{Z}_{2}$-theory [10].

In the Bose-Einstein condensate (BEC) of ultracold atoms, a similar method of formation of superfluid vortices has been studied [11-13]. In these studies, vortexantivortex pairs have been produced by periodically varying the trapping potential of the condensate. These studies suggest that under these periodic perturbations timedependent excitations arise in the condensate modifying the coherent wave function of the BEC. These excitations lead to the formation of vortex-antivortex pairs in the system. Subsequently, a tangled network of vortices is formed in the system, indicating a transition to a state of quantum turbulence. Ultimately, the system reaches a disordered state where BEC is completely destroyed. In these studies, the oscillation frequency of trapping potential is considered to be $200 \mathrm{~Hz}$, and the timescale for the whole process is a few tens of milliseconds. This timescale 
depends upon the amplitude and frequency of oscillations of trapping potential (i.e., rate of injected energy) and the energy of vortex configuration in the system.

Following the above studies in Refs. [9-13], it is an obvious question to ask if spacetime oscillations can also induce parametric resonance or large oscillations in a field. Such a question is relevant for many systems, for example, an ultralight axionlike field coupled to gravitational waves, neutron star superfluidity under timedependent tidal deformations, etc. During a binary neutron stars (BNS) merger, the orbiting neutron stars exert a time-dependent tidal force on each other [14]. The frequency and amplitude of this tidal force keep on increasing with time and become maximum toward the end of merger. Under such tidal force, depending upon the tidal deformability, the neutron star undergoes timedependent deformations. These tidal deformations can couple to the condensate of interior superfluid of the neutron star and may lead to the turbulence (formation of tangled vortices) as discussed in Refs. [11,12] (for the discussion on the possibility of superfluidity inside neutron star, see Refs. [15-17]). A similar kind of scenario, i.e., the occurrence of superfluid turbulence, superfluid to normal fluid transition, and glitch (or antiglitch) in neutron stars during BNS merger, has been discussed in Ref. [18] as well.

It has been studied in Ref. [19] that, due to the tidal force during the merger, energy pumped into the system is so high that the (local) temperature and density of neutron stars can reach up to $100 \mathrm{MeV}$ and a few times the nuclear saturation density, respectively. Therefore, in such a situation, neutron superfluidity can be destroyed (in some local regions) even before the completion of merger as the transition temperature for such superfluidity is somewhere in between 0.1 to $5 \mathrm{MeV}$ [20]. Therefore, the generation of excitations, vortex-antivortex pairs, and quantum turbulence in superfluid during the merger are expected to occur in the intermediate stage of evolution of neutron stars. It is also possible that the condensate excitation increases so much that the neutron star superfluidity is destroyed even before the transition temperatur is reached $[11,12])$. Certainly, the superfluid phase of quark matter (e.g., colorflavor locked phase) in the inner core of neutron stars can survive for a longer time as it has transition temperature approximately $100 \mathrm{MeV}$ [17].

To study the time evolution of interior superfluidity of the neutron star under such tidal deformations, one must solve the relativistic Gross-Pitaevskii equation, known as the Gross-Pitaevskii-Anandan equation [21], in the background of the spacetime metric of star with a timedependent perturbation appropriate for the BNS merger system. This also can be done by solving the nonlinear Klein-Gordon equation in the presence of an appropriate metric background as superfluidity can also be described in the field theoretical framework, where BEC is generally characterized by spontaneous breaking of U(1) global symmetry for a complex scalar field $[22,23]$.

In this paper, we study the effects of spacetime oscillations on the evolution of a complex scalar field. This may shed some light on the time dynamics of interior superfluidity of neutron star during merger. However, in this work, we are not doing phenomenology for any particular system. Rather, the present work is completely formal, where we show for the first time that a complex scalar field can be excited and topological vortices can be formed under the spacetime oscillations. Therefore, here, instead of considering a spacetime metric of a star, for simplicity, we take Minkowski metric with a periodic perturbation in time. This simplification helps to give a clear understanding of the effect of metric oscillations on the field and production of topological vortices. In this metric background, we numerically solve the nonlinear Klein-Gordon equation in $(2+1)$ dimensions for a complex scalar field with an initial field configuration given by $\Phi(x, y)=\Phi_{0}+\delta \Phi(x, y)$, where $\Phi_{0}$ is the vacuum expectation value (VEV) of U(1) symmetry broken effective potential and $\delta \Phi(x, y)$ represents small fluctuations of field about the VEV. Here, the initial field fluctuations have been considered as the spacetime oscillations couple with the field equation only through the spacetime derivatives of the field; see the equation of motion below.

We see that, under the spacetime oscillations, the field undergoes large amplitude oscillations for a wide range of frequencies of spacetime oscillations, which eventually leads to the formation of vortex-antivortex pairs. In the low-frequency regime (frequency smaller than the mass of field), mainly transverse modes (Goldstone modes) arise, while in the high-frequency regime, longitudinal modes also get generated dominantly. A detailed analysis shows that there is a correspondence between the frequency of spacetime oscillations and momentum of time growing field modes. This suggests that the field undergoes parametric resonance under the spacetime oscillations.

We mention that our present study is fundamentally different from the previous studies in Refs. $[9,10]$, even though the underlying physics for the generation of topological defects is the same, i.e., parametric resonance. In previous studies, oscillating temperature couples to the magnitude of the field. Therefore, to observe parametric resonance, the frequency of temperature oscillations must be close to the mass of longitudinal modes of the field. Whereas, in the present case, the spacetime oscillations couple to the gradient of field. This makes a direct coupling between the frequency of spacetime oscillations and the momentum of field modes present in the initial field configuration. Therefore, by satisfying the resonance condition, some specific field modes grow exponentially with time. This can occur, as long as the appropriate momentum modes of the field are present in the system. Thus, in the 
present case, the mass of the field does not set the lowestfrequency cutoff to induce parametric resonance; hence, vortex-antivortex pairs could be formed for a wide range of frequencies of spacetime oscillations. However, for this phenomenon, the lowest-frequency cutoff is set by the system size due to finite size effects. Our results suggest that the frequency of spacetime oscillations must be greater than $2 /$ (system size) to induce the parametric resonance of the field.

In the case of neutron stars during BNS merger, the accessible frequencies of spacetime oscillations are many orders of magnitude smaller than the mass of condensate field of interior superfluidity (approximately $0.1-5.0 \mathrm{MeV}$ ). With these frequencies, even the transverse modes of condensate field may have difficulties growing due to finite size effects. Our present results suggest that, in a typical neutron star of radius approximately $10 \mathrm{~km}$, to generate condensate excitations the frequency of spacetime oscillations must be greater than approximately $30 \mathrm{kHz}$. This required frequency is way beyond the reach of any known BNS merger systems, which could generate maximum frequency of spacetime oscillations up to approximately $1 \mathrm{kHz}$. Therefore, it is difficult to generate condensate excitations of neutron star superfluidity, under the phenomenon of parametric resonance, during BNS merger.

However, this has to be investigated in detail that whether there is any other method by which condensate excitation in neutron star superfluidity can be generated during mergers. We mention that in the case of BEC of ultracold atoms a $200 \mathrm{~Hz}$ frequency of oscillations of trapping potential is sufficient to excite the condensate with system size of only few hundred micrometers in a few tens of milliseconds time [11]. Therefore, it is not very unrealistic to expect the generation of such condensate excitations and formation of vortex-antivortex pairs in the interior superfluidity of neutron star during BNS merger. This requires a detailed investigation, which we will try to pursue in the future.

This paper is organized as follows. In Sec. II, we derive the equation of motion for a complex scalar field in the presence of oscillating spacetime metric background. In Sec. III, we outline the details of our numerical simulations. Then, we present our simulation results in Sec. IV. Finally, we briefly summerize our work and discuss future directions in Sec. V.

\section{EQUATION OF MOTION}

To study the effects of spacetime oscillations on a complex scalar field, we consider the spacetime metric as a periodic perturbation on the top of Minkowski metric. We consider the inverse spacetime metric as $g^{\mu \nu} \equiv$ $\operatorname{diag}(-1,1-\varepsilon \sin (\omega(t-z)), 1+\varepsilon \sin (\omega(t-z)), 1)$, where $\varepsilon(<1)$ and $\omega$ are the amplitude and frequency of spacetime oscillations, respectively; $(t, x, y, z)$ are spacetime coordinates. Note that this form of the metric has been chosen just for a simplicity in the equation of motion of the field, though any periodic time-dependent metric can be taken for our study. The action of the complex scalar field on the spacetime manifold with the given metric is $[23,24]$

$$
S=\int d^{4} x \sqrt{-g}\left[-\frac{1}{2} g^{\mu \nu} \partial_{\mu} \Phi^{*} \partial_{\nu} \Phi-V\left(\Phi^{*} \Phi\right)\right],
$$

where $g=\operatorname{det}\left(g_{\mu \nu}\right)=-\left(1-\varepsilon^{2} \sin ^{2}(\omega(t-z))\right)^{-1}, \Phi=\phi_{1}+$ $i \phi_{2}, \Phi^{*}=\phi_{1}-i \phi_{2} ; \phi_{1}$ and $\phi_{2}$ are the real scalar fields. We consider the symmetry breaking effective potential

$$
V\left(\Phi^{*} \Phi\right)=\frac{\lambda}{4}\left(\Phi^{*} \Phi-\Phi_{0}^{2}\right)^{2},
$$

where $\lambda$ is the self-interaction coupling parameter of the field and $\Phi_{0}$ is the VEV of the effective potential. With this, the mass of longitudinal component of the field is given by $m_{\Phi}=\Phi_{0} \sqrt{2 \lambda}$. The equations of motion for $\left(\phi_{1}, \phi_{2}\right)$ fields are [23,24]

$$
\square \phi_{i}-\frac{d V}{d \phi_{i}}=0,
$$

where $i=1,2$. The covariant d'Alembertian is given by

$$
\square \phi_{i}=\frac{1}{\sqrt{-g}} \partial_{\mu}\left(\sqrt{-g} g^{\mu \nu} \partial_{\nu} \phi_{i}\right) .
$$

Therefore, in the expanded form, the field equations become

$$
\begin{aligned}
& -\frac{1}{2} \frac{\varepsilon^{2} \omega \sin (2 \omega(t-z))}{\left(1-\varepsilon^{2} \sin ^{2}(\omega(t-z))\right)}\left(\frac{\partial \phi_{i}}{\partial t}+\frac{\partial \phi_{i}}{\partial z}\right)-\frac{\partial^{2} \phi_{i}}{\partial t^{2}} \\
& +(1-\varepsilon \sin (\omega(t-z))) \frac{\partial^{2} \phi_{i}}{\partial x^{2}}+(1+\varepsilon \sin (\omega(t-z))) \frac{\partial^{2} \phi_{i}}{\partial y^{2}} \\
& +\frac{\partial^{2} \phi_{i}}{\partial z^{2}}-\lambda \phi_{i}\left(\phi_{1}^{2}+\phi_{2}^{2}-\Phi_{0}^{2}\right)=0 .
\end{aligned}
$$

For the simplicity of solving it numerically, (i) we assume that there is no variation of the field $\Phi$ along the $z$ direction, and (ii) we look at the solution of the field only in the $z=0$ plane. With these simplifications, the above equations reduce to

$$
\begin{aligned}
& -\frac{1}{2} \frac{\varepsilon^{2} \omega \sin (2 \omega t)}{\left(1-\varepsilon^{2} \sin ^{2}(\omega t)\right)} \frac{\partial \phi_{i}}{\partial t}-\frac{\partial^{2} \phi_{i}}{\partial t^{2}}+(1-\varepsilon \sin (\omega t)) \frac{\partial^{2} \phi_{i}}{\partial x^{2}} \\
& +(1+\varepsilon \sin (\omega t)) \frac{\partial^{2} \phi_{i}}{\partial y^{2}}-\lambda \phi_{i}\left(\phi_{1}^{2}+\phi_{2}^{2}-\Phi_{0}^{2}\right)=0 .
\end{aligned}
$$

It is very clear from the above equations that the spacetime oscillations only couple to the derivatives of the field, where the coefficient of the first-order time derivative term oscillates with a mixture of frequencies $\omega$ and $2 \omega$. 
Under the spacetime oscillations, the gradient of the field oscillates, which must induce oscillations in the field via the parametric resonance with different possible oscillation frequencies. These field oscillations must be modulated by the steep rise of the effective potential in the longitudinal direction. The parametric resonance of the field eventually leads to the formation of vortex-antivortex pairs in the system. Our simulation results validate these expectations, which we present in the following sections.

\section{SIMULATION DETAILS}

To perform the simulation, we discretize the $x y$-plane into $200 \times 200$ lattice points with the lattice spacing of $\Delta x=\Delta y=0.01 \Lambda$, which gives the total lattice size $L=$ $2.0 \Lambda$ in each direction (here, $\Lambda$ is a unit of length and time, which we have not specified, as in this work we are not presenting phenomenological prediction for any system). $\Delta x$ and $\Delta y$ should be smaller than inverse of the field mass, i.e., $\Delta x, \Delta y<m_{\Phi}^{-1}$, so that a reasonable spatial variation of the field can be resolved. The time is discretized in the time step of $\Delta t=0.005 \Lambda$. Note that in the equation of motion [Eq. (6)] the discretization in the time axis works only if the condition $\Delta t<\omega^{-1}$ is satisfied. In our simulation, we study the effects of spacetime oscillations, in particular, the role of parameters $\varepsilon$ and $\omega$ on the field evolution. To describe the effects, for most of the simulations, we consider $\varepsilon=0.4$ and $\omega=100 \Lambda^{-1}$. We take the parameters of the effective potential as $\Phi_{0}=10 \Lambda^{-1}$ and $\lambda=40$. We also study the effects of changing these parameters on simulation results.

In Eq. (6), the field evolution is coupled with spacetime oscillations through a second-order spatial derivative and first-order time derivative of the field. Therefore, initial field configuration can respond to the spacetime oscillations only when fluctuations of the field (spacetime gradient) are present in the system, i.e., iff the field configuration is not in the complete minimum energy configuration. These fluctuations can naturally arise due to the presence of thermal and/or quantum fluctuations in the system. For simplicity, we consider fluctuations only in the initial field configuration; i.e., we take $\Phi(x, y)=$ $\Phi_{0}+\delta \Phi(x, y)$ at time $t=0$ and further evolve the field with Eq. (6).

In our simulation, for the initial field configuration, we have considered small spatial variations in $\phi_{1}$ and $\phi_{2}$ fields such that the magnitude of field $\Phi$ remains $\Phi_{0}$; i.e., the field is taken at the minima of the effective potential [Eq. (2)], and only transverse fluctuations of the field have been considered for the simulation. However, we have seen that considering fluctuations in the magnitude of the field does not change any qualitative aspect of the simulation results. More specifically, we have taken $\phi_{1}(x, y, t=0)=\Phi_{0} \sqrt{1-\alpha_{x y}^{2}}$ and $\phi_{2}(x, y, t=0)=\Phi_{0} \alpha_{x y}$, where $\alpha_{x y}$ varies randomly from point to point on the lattice in the range $[-\beta, \beta]$; here $0<\beta \ll 1$. This initial field configuration has a nonzero spatial gradient of both fields. In our simulation, we have taken $\beta=0.2$. We have seen that choosing different values of $\beta$ does not change our results qualitatively. We evolve this initial field configuration by solving Eq. (6) using the second-order Leapfrog method and considering periodic boundary condition along the spatial directions. By changing lattice size and boundary conditions, we have checked that the reflection of field fluctuations from the boundary has a negligible effect on our simulation results.

\section{SIMULATION RESULTS}

\section{A. Parametric resonance of field under spacetime oscillations}

We now present our simulation results. In Fig. 1, we show the vector plots of field configuration in the physical space ( $x y$ plane) at four different times of the field evolution. The $x$ and $y$ components of the vector field correspond to $\phi_{1}$ and $\phi_{2}$ fields, respectively. Note that the whole lattice area is $2.0 \times 2.0 \Lambda^{2}$, while only a small portion of the lattice is shown in the figure. In this simulation, we have taken $\varepsilon=0.4, \omega=100 \Lambda^{-1}$, $\Phi_{0}=10 \Lambda^{-1}$, and $\lambda=40$. As mentioned earlier that, only transverse fluctuations of the field have been considered in the initial field configuration, for which phase of the field varies randomly from one lattice point to other within the range of -0.2 to $0.2 \mathrm{rad}$ for the given value of $\beta$. Figure 1(a) shows such an initial field configuration, in which the phase fluctuations of field can be clearly seen. We observe that roughly during the time interval of $0.5-1.5 \Lambda$ the field itself starts performing oscillations in

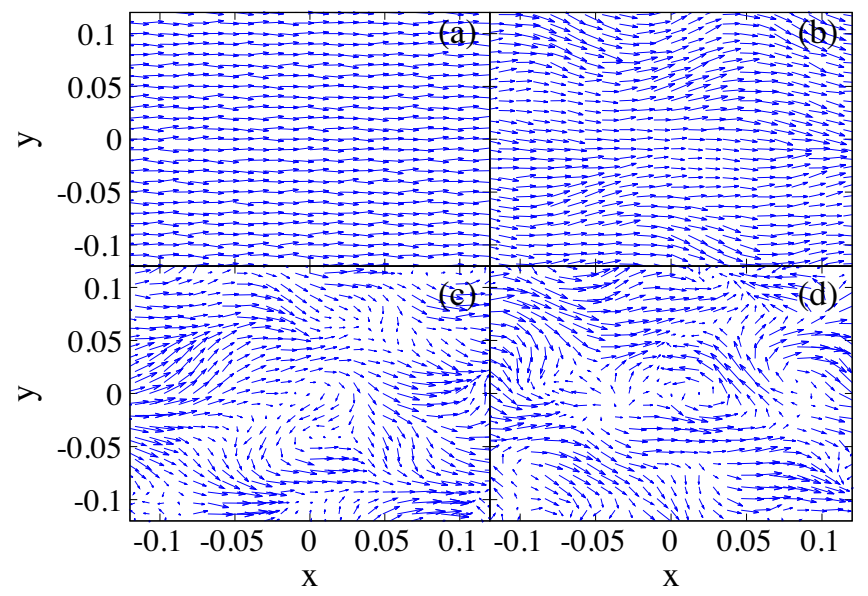

FIG. 1. The figure shows the vector plots of field configuration in physical space ( $x y$ plane) at four different times of field evolution. The parameters of simulation are $\varepsilon=0.4, \omega=100 \Lambda^{-1}$, $\Phi_{0}=10 \Lambda^{-1}$, and $\lambda=40$. Plots $\mathrm{a}, \mathrm{b}, \mathrm{c}$, and d correspond to the field configurations at times $t=0,1.35,1.7$, and $1.8 \Lambda$, respectively. In plot $\mathrm{d}$, vortex-antivortex pairs have been formed. Roughly at time $t=2.5 \Lambda$, the field configuration achieves a disordered state. 


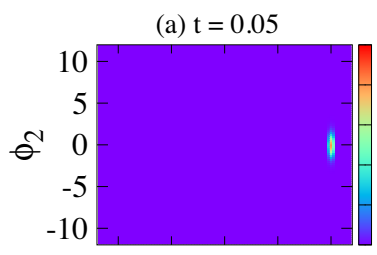

(c) $\mathrm{t}=1.35$
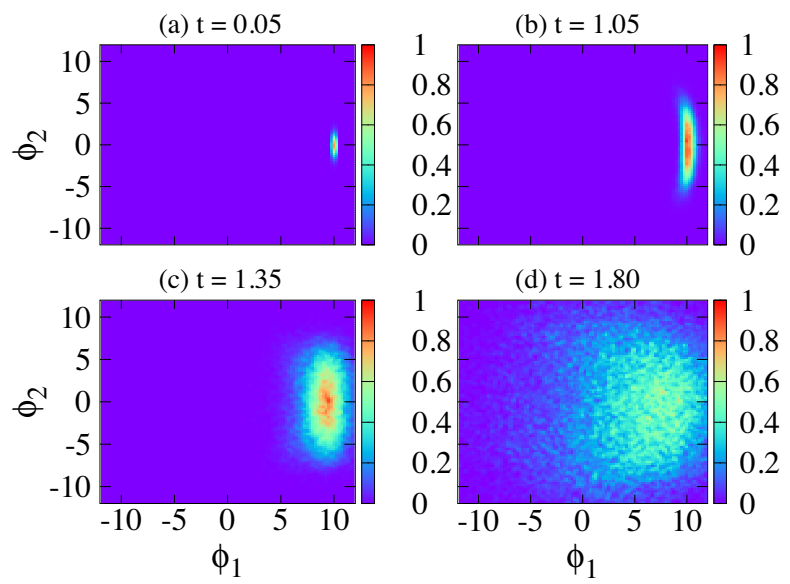

FIG. 2. In the figure, the distribution of field has been plotted in field space ( $\phi_{1} \phi_{2}$ plane) at four different times of field evolution. The distribution has been normalized such that in each plot density varies in between 0 and 1 . Plots a, b, c, and d correspond to the field distribution at times $t=0.05,1.05,1.35$, and $1.8 \Lambda$, respectively. In plot a, field distribution is highly localized about the chosen minima of the effective potential, which becomes more spread in plot $b$, where mostly transverse excitation has been generated. In plot $\mathrm{c}$, longitudinal excitation also has been generated. At the time stage of plot $d$, the field flips to the opposite side of the vacuum manifold, leading to the formation of vortex-antivortex pairs.

space and time with a significantly large amplitude; at time larger than this, the field dynamics becomes much more complicated. Figure 1(b) shows the field configuration at time $t=1.3 \Lambda$. This figure clearly shows that there is generation of a systematic periodic variation (waves) of the field in the physical space, whose amplitude keeps increasing with time within the time interval mentioned above. We observe that the wavelength of these waves is $\omega$ dependent; decreasing $\omega$ leads to increase in wavelength of these waves (see Figs. 4 and 5 in this regard). Depending upon the choice of $\omega$, these waves may contain transverse as well as longitudinal excitation of the field. Because of the generation of these excitations, the field acquires a significant energy in some parts of the physical space to climb the central barrier of the effective potential and fall into the opposite side of the vacuum manifold. This leads to the formation of vortex-antivortex pairs in the system. This process can be seen in Figs. 1(c) and 1(d), which are plotted at times $t=1.7 \Lambda$ and $t=1.8 \Lambda$, respectively. In this process, the vortex-antivortex pairs keep forming, and some of them annihilate with time, while the overall number of these pairs keeps increasing. Roughly at the time $t=2.5 \Lambda$, fluctuations become so strong that the field configuration no longer remains in the ordered state and achieves a disordered state (not shown in the figure).

In Fig. 2, we plot distribution of the field in the $\phi_{1} \phi_{2}$ plane (field space) at four different times of the field evolution. In this figure, the field has been mapped from the physical space to the field space, where the density of points has been indicated by colors. The distribution has been normalized such that in each plot density varies between 0 and 1 . The parameters of simulation are same as used in Fig. 1. Figure 2(a) shows the field distribution at time $t=0.05 \Lambda$. This shows a highly localized distribution of the field around $\phi_{1} \approx \Phi_{0}=10 \Lambda^{-1}, \phi_{2} \approx 0$ due to our initial choice of field values. In this figure, the maximum height of the distribution is 2672. As the field evolves, the distribution keeps spreading around the initial distribution in the field space. Figure 2(b) shows the field distribution at time $t=1.05 \Lambda$ with maximum distribution height of 316 . One can clearly see that at this time mostly the transverse excitation has been generated. Figure 2(c) shows the stage of field distribution at time $t=1.35 \Lambda$ with distribution height of 88. At this time, both types of excitations, transverse as well as longitudinal, have been generated in the system. Note that Figs. 2(b) and 2(c) correspond to the time duration in which the field oscillates with a significantly large amplitude, as shown in Fig. 1(b). These excitations ultimately lead to the formation of vortex-antivortex pairs in the system. The field distribution, at the time stage when these pairs have been formed, is shown in Fig. 2(d) at time $t=1.8 \Lambda$ with maximum distribution height of 26. Subsequently, the field distribution spreads over the field space symmetrically around $\phi_{1}=\phi_{2}=0$, the maximum extent of which becomes way beyond the VEV, signaling a disordered state of the field.

To see how fluctuations in fields $\phi_{1}$ and $\phi_{2}$ grow with time, we calculate field's fluctuations $\delta \phi_{i}(t)=\sqrt{\left\langle\phi_{i}^{2}(\vec{x}, t)\right\rangle-\left\langle\phi_{i}(\vec{x}, t)\right\rangle^{2}}$, where $i=1,2$, and brackets $\langle\ldots\rangle$ represent the volume average (areal average in two dimensions) of the field. Figure 3 shows the time evolution of $\delta \phi_{1}$ and $\delta \phi_{2}$ for two sets of parameters of the effective potential. The parameters of the spacetime metric for the simulations are $\varepsilon=0.4$ and $\omega=100 \Lambda^{-1}$, the same as used in Figs. 1 and 2. Plots a (blue) and b (gray) are the time evolution of $\delta \phi_{1}$ and $\delta \phi_{2}$ for $\Phi_{0}=10 \Lambda^{-1}$ and $\lambda=40$, while plots $\mathrm{c}$ (black) and $\mathrm{d}$ (red) are the time evolution of $\delta \phi_{1}$ and $\delta \phi_{2}$ for $\Phi_{0}=0.1 \Lambda^{-1}$ and $\lambda=4$, respectively. Note that $\phi_{1}$ and $\phi_{2}$ at initial time are proportional to $\Phi_{0}$, which is the reason $\delta \phi_{1}$ and $\delta \phi_{2}$ start with larger values in plots $\mathrm{a}$ and $\mathrm{b}$ in comparison with plots $\mathrm{c}$ and $\mathrm{d}$ even though the chosen value of $\beta$ for both sets of parameters of the effective potential is the same (indeed, the fluctuation in the phase of field $\Phi$ is the same for both the sets of parameters of the effective potential). The Fig. 3 clearly shows that in each case the growth of fluctuation in the $\phi_{2}$ field is larger than fluctuation in $\phi_{1}$, which is again due to our choice of initial field configuration. After some time, both the field fluctuations become degenerate. Note that there are oscillations in plots a and $\mathrm{b}$ during the time interval of $0.5-1.5 \Lambda$. These oscillations correspond to the large amplitude field oscillations as discussed in Fig. 1. 


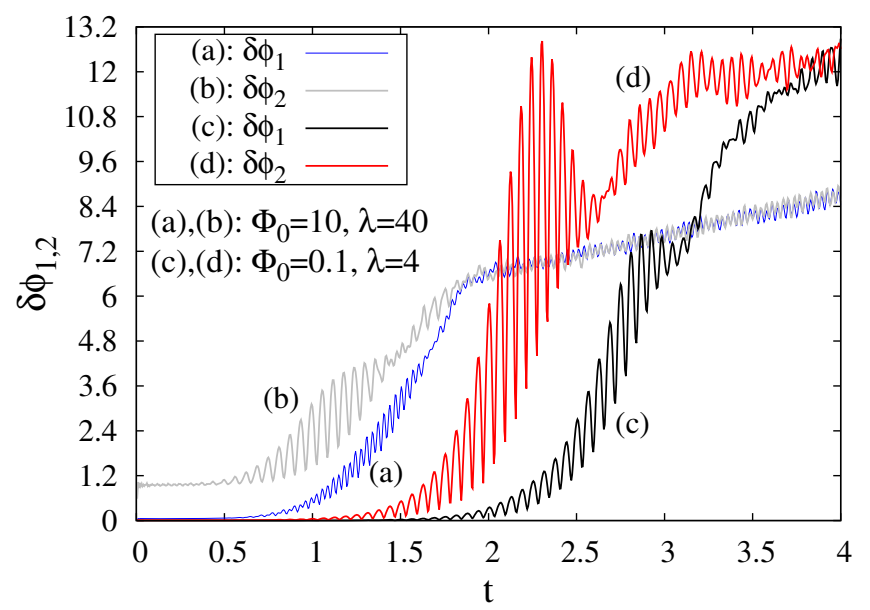

FIG. 3. The figure shows the time evolution of fluctuations $\delta \phi_{1}$ and $\delta \phi_{2}$ of fields $\phi_{1}$ and $\phi_{2}$, respectively, under the spacetime oscillations for two sets of parameters of the effective potential. The parameters of the spacetime metric are $\varepsilon=0.4$ and $\omega=100 \Lambda^{-1}$. Plots a (blue) and b (gray) are the time evolution of $\delta \phi_{1}$ and $\delta \phi_{2}$ for $\Phi_{0}=10 \Lambda^{-1}$ and $\lambda=40$, while plots c (black) and d (red) are for $\Phi_{0}=0.1 \Lambda^{-1}$ and $\lambda=4$, respectively. The figure clearly shows that in each case the initial growth of $\delta \phi_{2}$ is larger in comparison with $\delta \phi_{1} ; \delta \phi_{1}$ and $\delta \phi_{2}$ ultimately become degenerate. There are oscillations in plots a and $\mathrm{b}$ during the time interval of $0.5-1.5 \Lambda$, which correspond to the large amplitude field oscillations discussed in Fig. 1.

We now discuss a very crucial issue regarding the properties of formed vortices at different frequency regimes of spacetime oscillations. For two sets of parameters of the effective potential in Fig. 3, the masses of the field are $m_{\Phi}=89.44 \Lambda^{-1}$ and $m_{\Phi}=0.28 \Lambda^{-1}$, respectively. Therefore, with the used frequency $\omega$ in this figure, for the former case, $m_{\Phi}<\omega<2 m_{\Phi}$, while for the latter case, $\omega \gg m_{\Phi}$. Even though in both the cases there are qualitative similar growths in the fluctuations, there is a basic difference in the evolution of the field for these two cases. In the case of $\omega \gg m_{\Phi}$, spacetime oscillations generate waves in the field configuration having much shorter wavelength in comparison with $m_{\Phi}^{-1}$ (spacetime oscillations generate transverse modes with momentum $\approx \omega / 2$; see details later). Furthermore, later we show that for high frequency of spacetime oscillations, the longitudinal modes of the field also grow along with transverse modes following the relation $|\vec{k}|^{2}+m_{\Phi}^{2}=\left(\frac{n \omega}{2}\right)^{2}$, where $|\vec{k}|$ is the momentum of the longitudinal modes of the field, and $n=1,2,3, \ldots$. Therefore, in the case of $\omega \gg m_{\Phi}$, the fastest growing longitudinal modes (modes for $n=1$ and $n=2$ ) must have much larger momentum in comparison with $m_{\Phi}$. All this leads to huge excitations in the field for $\omega \gg m_{\Phi}$, and the field acquires value much larger than the VEV in a short time. In such a situation, the field does not feel the presence of the central barrier of the effective potential and does go back and forth across this easily.
As the core size of a topological vortex is given by $m_{\Phi}^{-1}$ where the field magnitude at distances larger than $m_{\Phi}^{-1}$ is given by the VEV, under such a huge field excitations having much shorter wavelength in comparison with $m_{\Phi}^{-1}$, there is no possibility of the formation of topological vortices. Thus, in the case of $\omega \gg m_{\Phi}$, topological vortices cannot be formed under the spacetime oscillations. Note that for the second set of parameters of the effective potential, our lattice size is smaller than $m_{\Phi}^{-1}$; hence, it cannot accommodate even a single topological vortex for these parameters. However, our above conclusion holds true even for a lattice whose size is much bigger than $m_{\Phi}^{-1}$.

However, in this case with sufficient evolution of the field, after time $t \simeq 2.7 \Lambda$, we see the formation of some vortex-antivortex kind of structures (even on the used lattice), though these are not topological vortices. In these vortices, due to huge excitations, the field acquires values way beyond the VEV outside the vortex core; note that in this case the VEV is $0.1 \Lambda^{-1}$, where outside these vortices, at this time, field acquires value $\simeq 20 \Lambda^{-1}$, which is 200 times larger than the VEV. Furthermore, in this case, we have found that the vortex core size is given by $\omega^{-1}$ (instead of $m_{\Phi}^{-1}$ ), as expected from the wavelength of the generated field excitations. Thus, these vortices certainly do not satisfy the properties of topological vortices; rather, their properties are determined by the parameters of spacetime oscillations. Basically, in this case, the magnitude of the field becomes so large in comparison with the VEV that the field configuration is able to create vortices (nontopological) with a core size of a much shorter length scale than $m_{\Phi}^{-1}$. (We show the vortices in the case of $\omega \gg m_{\Phi}$ in Fig. 14, where $m_{\Phi}=0.28 \Lambda^{-1}$ and $\omega=17 \Lambda^{-1}$ have been used for the simulation).

On the other hand, in the former case as discussed above, although longitudinal excitation arises and hence vortices are not well formed, vortices up to some extent satisfy the properties of topological vortices; see Figs. 1 and 2. Later, we show that there is a fundamental difference in the evolution of field for $\omega>m_{\Phi}$ and for $\omega \leq m_{\Phi}$. In the case of $\omega \leq m_{\Phi}$, the field remains close to the VEV where mainly transverse excitation arises under the spacetime oscillations. Therefore, the formed vortices in such a case certainly are topological vortices; see Fig. 10. Thus, only in those cases, for which frequency is either less than or close to the mass of the field, i.e., for which $\omega \lesssim m_{\Phi}$, the formed vortices under the spacetime oscillations are topological in nature. In our simulations, however, the field profile of these vortices never achieves an exact profile of topological vortices as the field configuration has to bear continuous spacetime oscillations.

To further investigate the behavior of field configurations at various time stages, we calculate the time evolution of various momentum modes of the fields $\phi_{1}$ and $\phi_{2}$. The Fourier transform of the field configuration from 

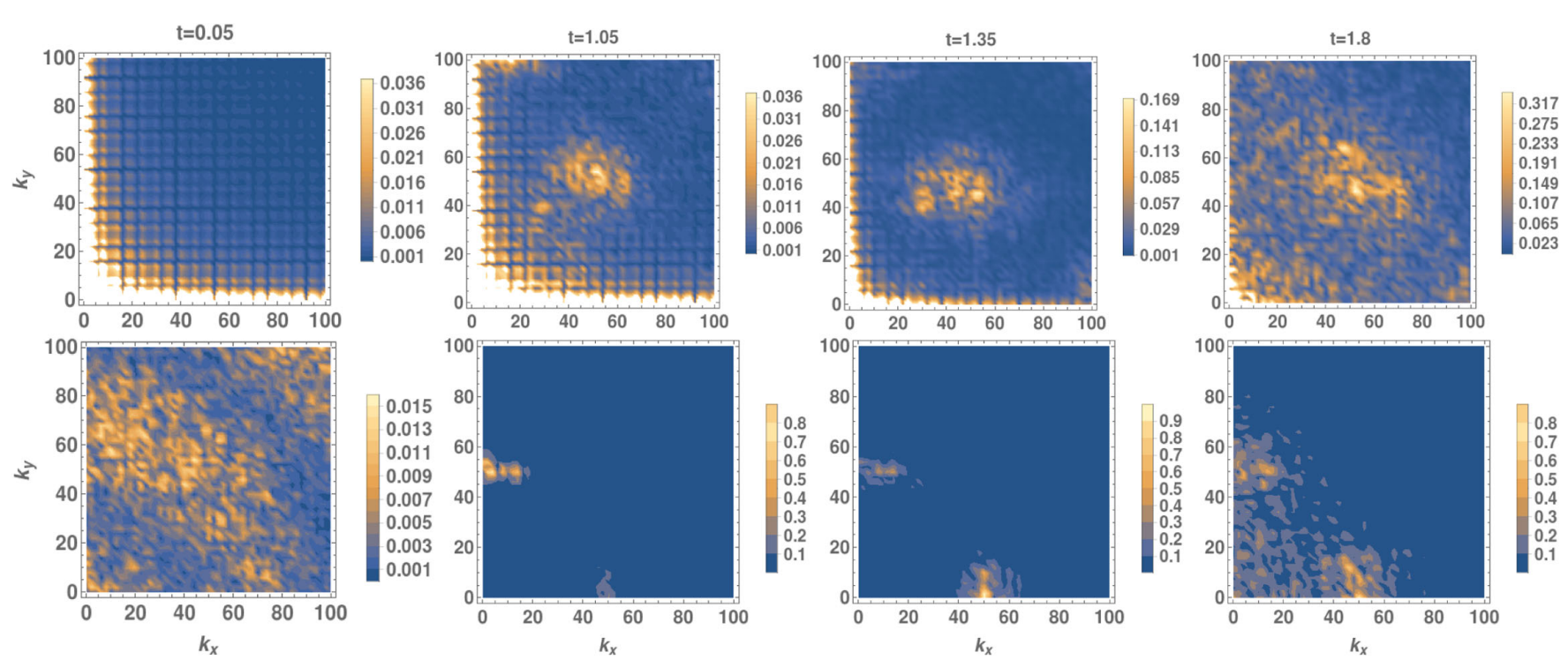

FIG. 4. The figure shows the profile of modulus of field modes $\left|\tilde{\phi}_{1}(\vec{k}, t)\right|$ (upper panel) and $\left|\tilde{\phi}_{2}(\vec{k}, t)\right|$ (lower panel) at different times for $\omega=100 \Lambda^{-1}, \varepsilon=0.4, \Phi_{0}=10 \Lambda^{-1}$, and $\lambda=40$. From left to right, modes are plotted at times $t=0.05,1.05,1.35,1.8 \Lambda$. There is noticeable growth of $\left|\tilde{\phi}_{1}(\vec{k}, t)\right|$ at $k_{x}=15 \Lambda^{-1}, k_{y}=\omega$ and $k_{x} \approx k_{y} \approx \sqrt{\left(\omega^{2}-\left(\frac{m_{\Phi}}{\sqrt{2}}\right)^{2}\right) / 2}=54.77 \Lambda^{-1}$, and growth of $\left|\tilde{\phi}_{2}(\vec{k}, t)\right|$ at $k_{x}=0, k_{y}=\omega / 2$ and $k_{x}=\omega / 2, k_{y}=0$. The subsequent evolution also leads to the growth of various other momentum modes of both fields.

the physical space to the momentum space at any time is given by

$$
\tilde{\phi}_{i}(\vec{k}, t)=\frac{1}{\mathcal{A}} \int_{\text {b.c. }} d^{2} \vec{x} \phi_{i}(\vec{x}, t) e^{i \vec{k} \cdot \vec{x}} ; \quad i=1,2
$$

where $\mathcal{A}$ is the total area of the system, b.c. stands for the boundary condition, and $\vec{k}=k_{x} \hat{x}+k_{y} \hat{y}, \quad \vec{x}=x \hat{x}+y \hat{y}$ are the momentum and position vectors, respectively. In Figs. 4 and 5, we plot the modulus of momentum modes of fields $\phi_{1}$ and $\phi_{2}$, i.e., $\left|\tilde{\phi}_{1}(\vec{k}, t)\right|$ (upper panel) and $\left|\tilde{\phi}_{2}(\vec{k}, t)\right|$ (lower panel), in the momentum space with spacing $\Delta k_{x}=\Delta k_{y}=2.0 \Lambda^{-1}$. (The white regions in the plots correspond to the magnitude larger than the maximum range of the legend.) In Fig. 4, we have taken spacetime oscillation frequency larger than $m_{\Phi}$, while in Fig. 5, the frequency is taken smaller than that. Therefore, the field dynamics is expected to be different in both the cases. Note that for the $\phi_{1}$ field, the zero mode (zero-momentum mode) is the most dominant mode, while for $\phi_{2}$, it is close to zero. This is just because of initially chosen values of these fields. In Fig. 4, we have taken $\omega=100 \Lambda^{-1}$, $\varepsilon=0.4, \Phi_{0}=10 \Lambda^{-1}$, and $\lambda=40$ for the simulation. From left to right, field modes are plotted at times $t=0.05,1.05,1.35,1.8 \Lambda$; see also Figs. 1 and 2 . Note that the time interval $0.5-1.5 \Lambda$ is the time stage of large amplitude field oscillations for the given $\omega$. At the initial time itself, all higher momentum modes of the field up to $k_{x}=k_{y}=2 \pi / \Delta x$ are present due to the prescribed random fluctuations, which can be seen in the plot at time $t=0.05 \Lambda$. Under the spacetime oscillations, depending upon the frequency $\omega$, some specific momentum modes of the field grow with time. In the figure, there is noticeable growth of $\left|\tilde{\phi}_{1}(\vec{k}, t)\right| \quad$ at $k_{x}=15 \Lambda^{-1}, \quad k_{y}=\omega$ and $k_{x} \approx k_{y} \approx \sqrt{\left(\omega^{2}-\left(\frac{m_{\Phi}}{\sqrt{2}}\right)^{2}\right) / 2}=54.77 \Lambda^{-1}$, and growth of $\left|\tilde{\phi}_{2}(\vec{k}, t)\right|$ at $k_{x}=0, k_{y}=\omega / 2$ and $k_{x}=\omega / 2, k_{y}=0$. The subsequent evolution also leads to the growth of various other momentum modes of both fields.

In Fig. 5, we have taken $\omega=50 \Lambda^{-1}, \varepsilon=0.4$, $\Phi_{0}=10 \Lambda^{-1}$, and $\lambda=40$ for the simulation. From left to right, field modes are plotted at times $t=2.0,2.5,3.0$, $3.5 \Lambda$. Note that for the given $\omega$ the time interval 2.0-3.7 $\Lambda$ corresponds to the stage of large amplitude oscillations of the field. In this case also, the initial distributions of field modes are the same as plotted in Fig. 4 at time $t=0.05 \Lambda$ (therefore, not shown again in Fig. 5). The figure clearly shows that there is noticeable growth of $\left|\tilde{\phi}_{1}(\vec{k}, t)\right|$ at $k_{x}=0$, $k_{y}=\omega$, and growth of $\left|\tilde{\phi}_{2}(\vec{k}, t)\right|$ at $k_{x}=0, k_{y}=\omega / 2$. In the intermediate stage of field evolution, there is also growth of $\left|\tilde{\phi}_{1}(\vec{k}, t)\right|$ at $k_{x} \approx \omega / 2, k_{y} \approx \omega / 2$ and $k_{x} \approx \omega / 2$, $k_{y} \approx 3 \omega / 2$. In this case also, the subsequent evolution leads to the growth of various other momentum modes of both fields.

To understand why some specific momentum modes of the field grow for a given $\omega$, we write the field as $\Phi=$ $\Phi_{0}+v_{1}+i v_{2}\left(\phi_{1}=\Phi_{0}+v_{1}, \phi_{2}=v_{2}\right)$, where $v_{1}$ and $v_{2}$ are small fluctuations of the field about the VEV. We write 

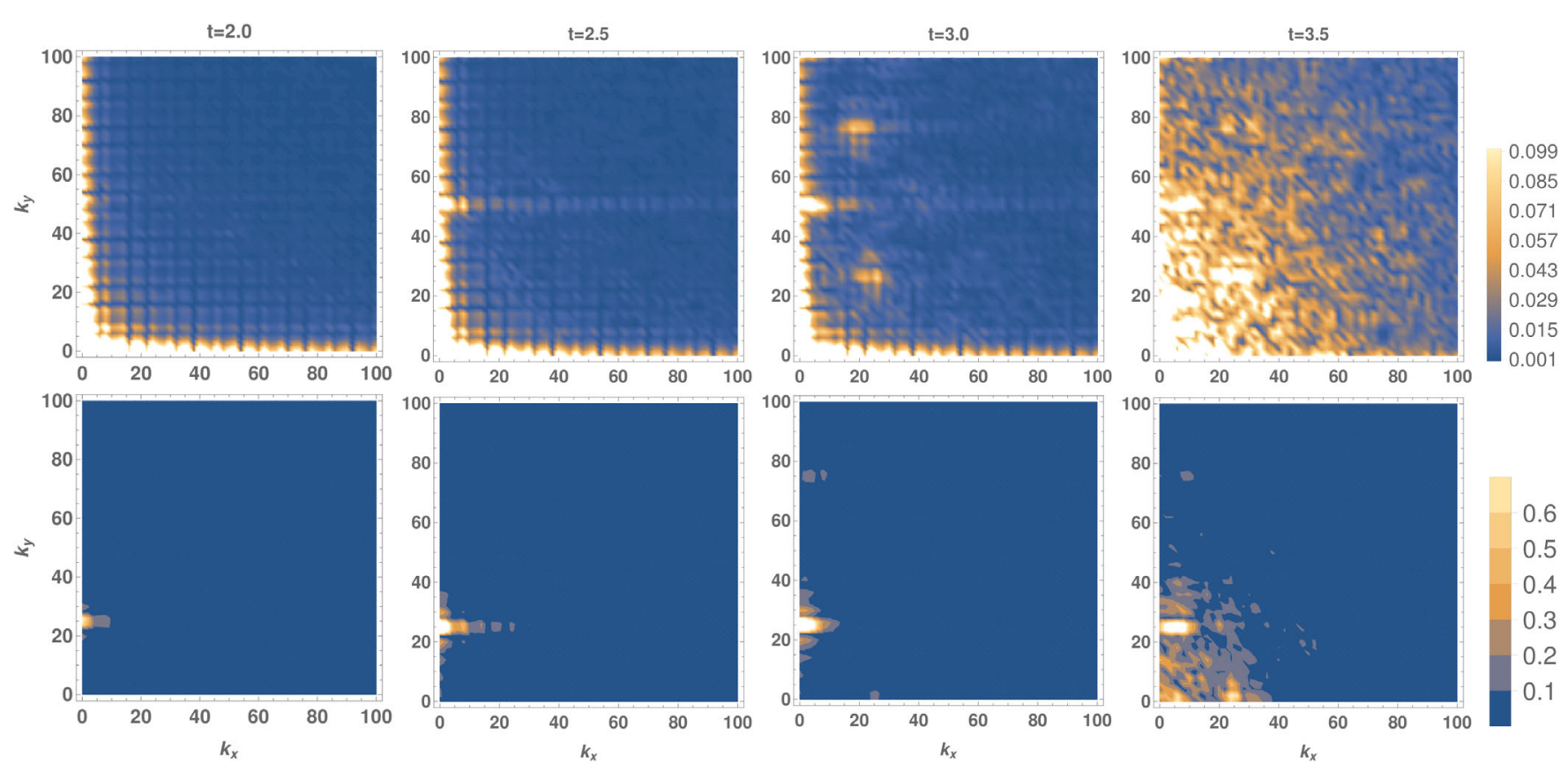

FIG. 5. The figure shows the profile of modulus of field modes $\left|\tilde{\phi}_{1}(\vec{k}, t)\right|$ (upper panel) and $\left|\tilde{\phi}_{2}(\vec{k}, t)\right|$ (lower panel) at different times for $\omega=50 \Lambda^{-1}, \varepsilon=0.4, \Phi_{0}=10 \Lambda^{-1}$, and $\lambda=40$. From left to right, modes are plotted at times $t=2.0,2.5,3.0,3.5 \Lambda$. There is noticeable growth of $\left|\tilde{\phi}_{1}(\vec{k}, t)\right|$ at $k_{x}=0, k_{y}=\omega$, and growth of $\left|\tilde{\phi}_{2}(\vec{k}, t)\right|$ at $k_{x}=0, k_{y}=\omega / 2$. In the intermediate stage of field evolution, there is also growth of $\left|\tilde{\phi}_{1}(\vec{k}, t)\right|$ at $k_{x} \approx \omega / 2, k_{y} \approx \omega / 2$ and $k_{x} \approx \omega / 2, k_{y} \approx 3 \omega / 2$. The subsequent evolution also leads to the growth of various other momentum modes of both fields.

the equation of motion for these fluctuations, keeping terms up to linear order in these fields, and then Fourier transform in the momentum space. The evolution equations for momentum modes of these fluctuations are

$\frac{\varepsilon^{2} \omega \sin (2 \omega t)}{2 f(t) f(-t)} \dot{\tilde{v}}_{i k}+\ddot{\tilde{v}}_{i k}+\left(k_{x}^{2} f(t)+k_{y}^{2} f(-t)+m_{i}^{2}\right) \tilde{v}_{i k}=0$,

where $\tilde{v}_{i k} \equiv \tilde{v}_{i}(\vec{k}, t), f(t)=1-\varepsilon \sin (\omega t), m_{1}=m_{\Phi}$, and $m_{2}=0$. These equations clearly show that $\tilde{v}_{1 k}$ and $\tilde{v}_{2 k}$ undergo parametric resonance (get resonant growths) if suitable conditions (relations between $k_{x}, k_{y}, m_{i}$, and $\omega$ ) are satisfied. We numerically solve these equations and find that there are various resonance conditions for both fields. In a reasonable range of frequency $\omega$, the resonance conditions for $\tilde{v}_{i k}$ are $|\vec{k}|^{2}+m_{i}^{2}=\left(\frac{n \omega}{2}\right)^{2}$, where $n=1,2,3, \ldots$. For each $n$, there are different frequency cutoff for $\tilde{v}_{1 k}$ to get resonance growth, which are $\omega \leq 2 m_{\Phi} / n$. This indicates that the most dominant modes of $\tilde{v}_{1 k}$, i.e., modes for $n=1$ and $n=2$, grow only when $\omega$ is greater than $m_{\Phi}$. Thus, effectively, the frequency cutoff for the longitudinal modes is $\omega \leq m_{\Phi}$. On the other hand, for $\tilde{v}_{2 k}$, there is no such frequency cutoff (due to its zero mass), therefore it can undergo parametric resonance even for any low frequency $\omega$ (where $\omega>0$ ). For a comparison, if one ignores the first term from the equation of $\tilde{v}_{2 k}$, then in
$(1+1)$ dimensions, it takes the form of a harmonic oscillator equation with the (time-dependent) frequency square $k_{x(y)}^{2}(1 \mp \sin (\omega t))$ [25]. In such a case, the oscillator undergoes parametric resonance when the condition $k_{x(y)} \simeq$ $n \omega / 2$ is satisfied [25]. For a given frequency $\omega$, the growth rates of growing modes of $\tilde{v}_{i k}$ are different and get changed by changing $\omega$. The fastest growing modes out of $\tilde{v}_{1 k}$ and $\tilde{v}_{2 k}$ are $\tilde{v}_{2 k}$ at $k_{x(y)}=\omega / 2, k_{y(x)}=0$, whose growth rates decrease by decreasing frequency of spacetime oscillations. This response of field modes to the frequency $\omega$ can play an important role in the understanding of the time of formation of first vortex-antivortex pair in the system at different frequencies $\omega$.

A similar feature of the resonance growths as discussed above can be seen in Figs. 4 and 5, where $\tilde{\phi}_{2}$ grows resonantly at $k_{x(y)} \simeq \omega / 2, k_{y(x)} \simeq 0$. In these figures, there is a qualitative difference in the evolution of $\tilde{\phi}_{1}$ for $\omega>m_{\Phi}$ and $\omega<m_{\Phi}$, as for $\omega>m_{\Phi}$ some momentum modes of the longitudinal component $\left(\tilde{v}_{1 k}\right)$ also grow resonantly. This shows that in the frequency regime of $\omega>m_{\Phi}$, both components of the field [transverse as well as longitudinal] are generated under the resonance process, whereas in the frequency regime of $\omega \leq m_{\Phi}$, a predominantly transverse component is generated. It has to be noted that in Fig. $4 \tilde{\phi}_{1}$ grows by following relation $|\vec{k}|^{2}+\left(\frac{m_{\Phi}}{\sqrt{2}}\right)^{2}=\omega^{2}$ instead of following the relation obtained for $\tilde{v}_{1 k}$ at $n=2$. This is happening probably because $\phi_{1}$ is the field whose values 
are given from the center of the effective potential. It is important to appreciate that the appropriate momentum modes of the field corresponding to $\omega$ have to be present initially to have resonance growths, where transverse modes of the field can grow even at frequencies less than the mass of the field. Thus, under the spacetime oscillations, there is no lowest-frequency cutoff for resonance to occur as long as the appropriate momentum modes of the field are present initially to grow (however, finite size effects of the system set a lowest-frequency cutoff; see discussions later). We point out that the above discussion is based on the linear approximation, while $\phi_{1}$ and $\phi_{2}$ are the coupled fields whose dynamics are modulated by the effective potential. This couples various momentum modes of the field nonlinearly and makes resonance conditions much more complicated.

The above analysis shows that the higher momentum modes of fields $\phi_{1}$ and $\phi_{2}$, following their respective resonance conditions, grow with time. Now, we show how zero modes (modes for $k_{x}=0, k_{y}=0$ ) of these fields vary with time. The zero modes of fields characterize the field configuration at each time and provide the frequency of oscillations during the large-amplitude oscillation stage of the field. Figure 6 shows the time evolution of the real part of zero modes of fields $\phi_{1}$ and $\phi_{2}$ for two sets of parameters of the effective potential. Note that the imaginary part of zero modes for both the fields is zero. In the figure, zero mode of the $\phi_{1}$ field is normalized with the respective $\Phi_{0}$ value. For this simulation, we have taken $\varepsilon=0.4$ and $\omega=100 \Lambda^{-1}$. Solid (blue) and dot-dashed (gray) lines are the time evolution of zero modes of fields $\phi_{1}$ and $\phi_{2}$, respectively, for $\Phi_{0}=10 \Lambda^{-1}, \lambda=40$. In this case, there are oscillations in the $\phi_{1}$ zero mode during the time interval of $0.5-1.5 \Lambda$ with growing and then decaying amplitude; let us call it the large amplitude field oscillation stage. Roughly at time $t=1.5 \Lambda$, these oscillations stop, and the zero mode starts collapsing toward the zero value. The collapse of the zero mode starts roughly at the time of formation of vortex-antivortex pairs in the system. We have performed the simulations for different spacetime oscillation frequencies and calculated the time evolution of the $\phi_{1}$ zero mode for each case (in the figure, it is not shown for other frequencies). We see that in all cases at initial time there is a unique frequency and amplitude of oscillations of the $\phi_{1}$ zero mode, which with subsequent evolution get modulated with a new frequency of oscillations with growing amplitude as shown in Fig. 6 from time $t=0.5 \Lambda$. This shows that the initial time oscillations of the $\phi_{1}$ zero mode (before time $t=0.5 \Lambda$ in Fig. 6) are independent from the spacetime oscillations and may be related with the evolution of initial field fluctuations. On the other hand, the new modulation frequency, i.e., the frequency during the time stage of large amplitude oscillations of the $\phi_{1}$ zero mode, is exactly equal to the spacetime oscillation frequency $\omega$ (which we show later).

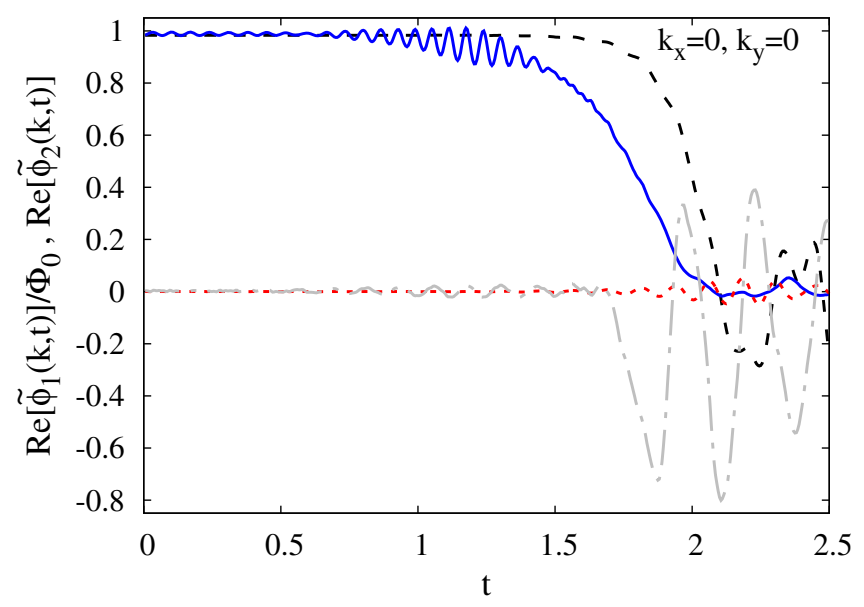

FIG. 6. The figure shows the time evolution of real part of zero modes of fields $\phi_{1}$ and $\phi_{2}$ for two sets of parameters of the effective potential. In the figure, the zero mode of the $\phi_{1}$ field is normalized with the respective $\Phi_{0}$ value. We have taken $\varepsilon=0.4$ and $\omega=100 \Lambda^{-1}$ for the simulation. Solid (blue) and dot-dashed (gray) lines are the time evolution of zero modes of fields $\phi_{1}$ and $\phi_{2}$ for $\Phi_{0}=10 \Lambda^{-1}$ and $\lambda=40$, while dashed (black) and dotted (red) lines are for $\Phi_{0}=0.1 \Lambda^{-1}$ and $\lambda=4$, respectively. Note that there are small amplitude oscillations in dashed (black) line, which does not appear because of the large $y$ range.

This gives more evidence of the parametric resonance of the field under the spacetime oscillations.

In Fig. 6, zero mode of the $\phi_{2}$ field [shown by dot-dashed (gray) line] also has small amplitude oscillations roughly in the same time interval (i.e., in time interval of $0.5-1.5 \Lambda$ ), but without much growth. Later, we show that the oscillation frequency of the zero mode of $\phi_{2}$ during this time interval is half the spacetime oscillation frequency. Here, it should be noted that during this time interval $\phi_{2}$ varies in between negative and positive values spatially as well as temporarily, as can be seen in Fig. 1(b). Therefore, even though the absolute value of $\phi_{2}$ reaches a nonzero value in some regions, its zero mode comes out to be very small (just because of the cancellation of positive and negative values). This is the reason why the zero mode of $\phi_{2}$ does not show any growth during this time interval (indeed, this mode does not follow the resonance condition). It is already clear from Fig. 3 that during this time interval the growth in the fluctuations of the field $\phi_{2}$ is larger in comparison with fluctuations in $\phi_{1}$. Therefore, the higher momentum modes of $\phi_{2}$ must show growths with time, which already has been seen in Fig. 4 at $k_{x}=\omega / 2, k_{y}=0$ and $k_{x}=0, k_{y}=\omega / 2$. In Fig. 7, we plot the time evolution of the real and imaginary parts of the $\left(k_{x}=\omega / 2, k_{y}=0\right)$ mode of $\phi_{2}$ with solid (red) and dotted (blue) lines, respectively. This clearly shows oscillations of these modes with growing and then decaying amplitude with time.

To show the dependence of the zero modes of fields $\phi_{1}$ and $\phi_{2}$ on the parameters of the effective potential, in 


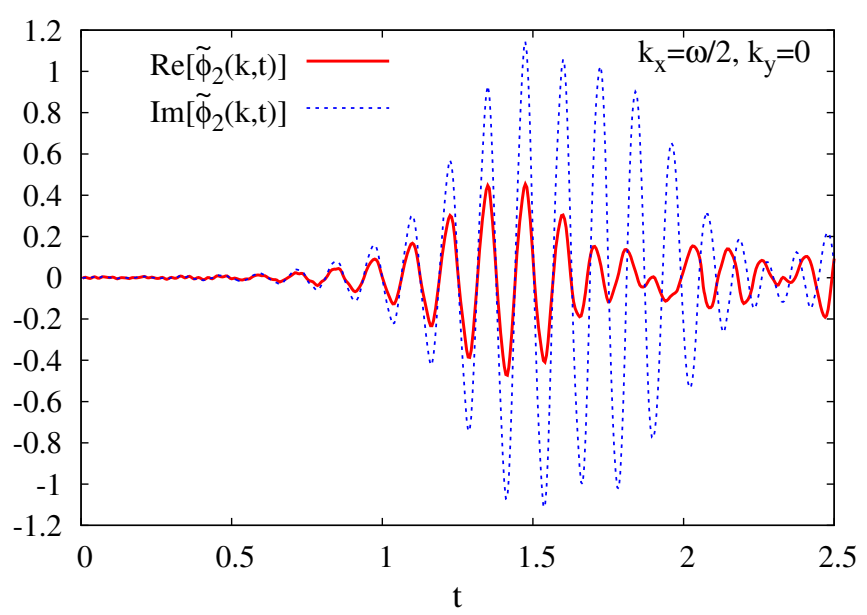

FIG. 7. The figure shows the time evolution of real and imaginary parts of the $\left(k_{x}=\omega / 2, k_{y}=0\right)$ mode of field $\phi_{2}$ with solid (red) and dotted (blue) lines, respectively, for $\omega=100 \Lambda^{-1}, \varepsilon=0.4, \Phi_{0}=10 \Lambda^{-1}$, and $\lambda=40$.

Fig. 6, we also plot their time evolution for $\Phi_{0}=0.1 \Lambda^{-1}$ and $\lambda=4$ with dashed (black) and dotted (red) lines, respectively. In this case, the depth of the effective potential is very small; therefore, the field can easily climb the central barrier at $\Phi=0$. Note that in this case the amplitude of oscillations of the $\phi_{1}$ zero mode is small, which is not clearly visible because of the large $y$ range in the plot. The amplitude of oscillations of the $\phi_{2}$ zero mode is also very small in the plot because of the same reason mentioned above.

To determine the oscillation frequency of fields during the time interval of $t_{i}-t_{f}$ in which the $\phi_{1}$ zero mode oscillates with a significantly large amplitude, we perform Fourier transform of the fields' zero mode from $t$ space to the frequency $f$ space as

$\Pi_{i}(\vec{k}, f)=\frac{1}{\left(t_{f}-t_{i}\right)} \int_{t_{i}}^{t_{f}} d t \tilde{\phi}_{i}(\vec{k}, t) e^{-i f t} ; \quad i=1,2$.

We calculate the moduli of $\Pi_{1}(\vec{k}, f)$ and $\Pi_{2}(\vec{k}, f)$, which provide the frequency spectrum of momentum modes of $\phi_{1}$ and $\phi_{2}$ fields. Figure 8 shows the frequency spectrum of the zero mode of $\phi_{1}$ during the time interval of $t_{i}-t_{f}$ for different spacetime oscillation frequencies $\omega$. In the figure, such a time interval for each frequency $\omega$ is mentioned in brackets. To obtain this frequency spectrum, in each case, $\Phi_{0}$ has been subtracted from the $\phi_{1}$ zero mode so that the background frequency modes, arising due to constant $\Phi_{0}$ value, get eliminated and the peak structures become apparent. (However, this subtraction does not remove background frequency modes completely, which is the reason a peak at the zero frequency is still present in each plot). In the figure, violet (dotted), green (solid thin), and black (solid thick) lines correspond to the frequency spectrum for $\omega=20 \Lambda^{-1}, \quad 50 \Lambda^{-1}$, and $100 \Lambda^{-1}$,

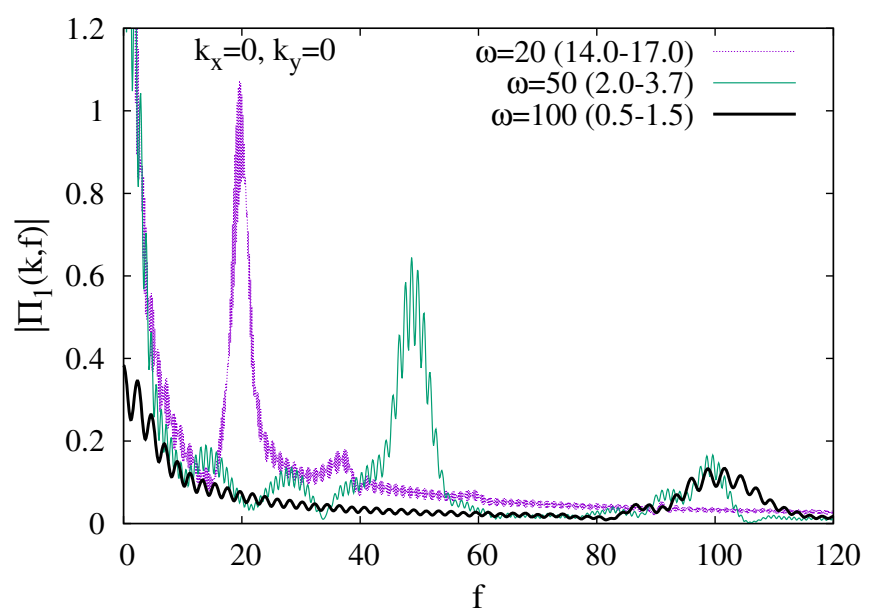

FIG. 8. The figure shows the frequency spectrum of the $\phi_{1}$ zero mode for different spacetime oscillation frequencies $\omega$ in the time interval in which it oscillates with a significant large amplitude. This time interval for the respective frequencies is written in brackets. Violet (dotted), green (solid thin), and black (solid thick) lines correspond to the frequency spectrum for $\omega=20,50$, and $100 \Lambda^{-1}$. In each case, the frequency spectrum has a peak at the respective frequency $\omega$ of spacetime oscillations, which indicates the phenomenon of parametric resonance of the field.

respectively. In each case, $\varepsilon=0.4, \Phi_{0}=10 \Lambda^{-1}$, and $\lambda=40$ have been taken for simulations. In the figure, in each case, the frequency spectrum has a peak at the respective frequency $\omega$ of spacetime oscillations, suggesting the phenomenon of parametric resonance. Note that, for the frequency $\omega=50 \Lambda^{-1}$, there are two peaks at $f=50 \Lambda^{-1}$ (dominant peak) and at $f=100 \Lambda^{-1}$ (subdominant peak). The second peak is arising maybe due to coefficient $\sin (2 \omega t)$ of the first-order time derivative term in Eq. (6) or maybe due to resonance process itself. For $\Phi_{0}=0.1 \Lambda^{-1}$ and $\lambda=4$, the amplitude of oscillations of the $\phi_{1}$ zero mode is too small to determine such peak structure, which is why the frequency spectrum for this case has not been shown in the figure.

Figure 9 shows the frequency spectrum of the zero mode of $\phi_{2}$ in the same time interval as mentioned in Fig. 8 for different spacetime oscillation frequencies $\omega$ (the time interval $t_{i}-t_{f}$ for each frequency $\omega$ is mentioned in brackets). Unlike for the $\phi_{1}$ zero mode, in this case, there is no background frequency spectrum. In the figure, violet (dotted), green (solid thin), and black (solid thick) lines correspond to the frequency spectrum for $\omega=20 \Lambda^{-1}$, $50 \Lambda^{-1}$, and $100 \Lambda^{-1}$, respectively. In this case, each frequency spectrum has peak at half the spacetime oscillation frequency $\omega$. For $\omega=20 \Lambda^{-1}$, there is no unique and strong peak structure in the frequency spectrum. In the figure, the red (dashed) line corresponds to the case for $\omega=100 \Lambda^{-1}, \Phi_{0}=0.1 \Lambda^{-1}$, and $\lambda=4.0$. In this case also, the frequency spectrum has a peak at the 


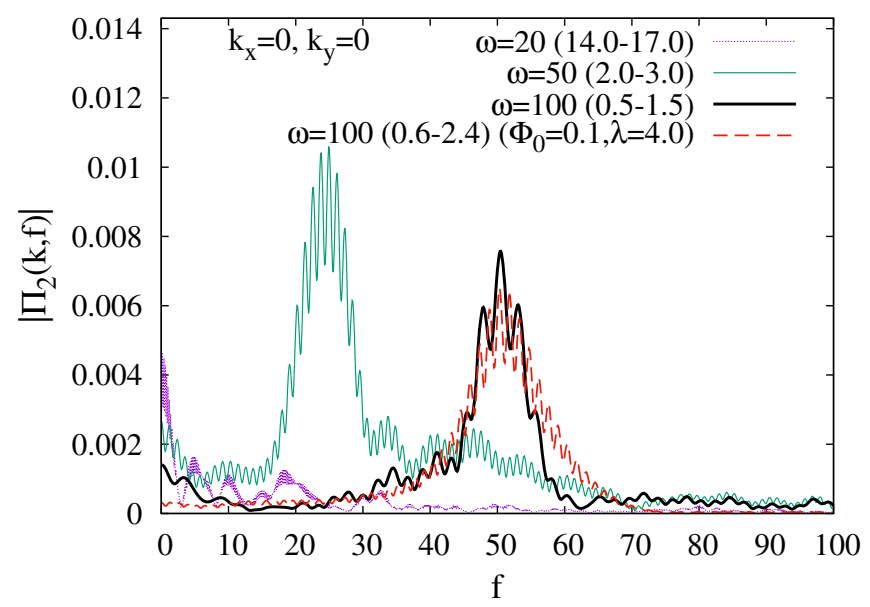

FIG. 9. The figure shows the frequency spectrum of the $\phi_{2}$ zero mode for different spacetime oscillation frequencies $\omega$ in the same time interval as mentioned in Fig. 8 (mentioned here as well in brackets). Violet (dotted), green (solid thin), and black (solid thick) lines correspond to the frequency $\omega=20,50$, and $100 \Lambda^{-1}$. The peak structures show that the zero mode of $\phi_{2}$ oscillates with frequency $\omega / 2$. The red (dashed) line corresponds to the case for $\omega=100 \Lambda^{-1}$, $\Phi_{0}=0.1 \Lambda^{-1}$, and $\lambda=4$, which also has a peak at $\omega / 2$.

half $\omega$, which shows the independence of some aspects of this phenomenon from the parameters of the effective potential. We have also checked that the real and imaginary parts of momentum modes of $\phi_{2}$ at $k_{x}=\omega / 2, k_{y}=0$ and $k_{x}=0, k_{y}=\omega / 2$ (see Fig. 7) also oscillate with frequency $\omega / 2$.

With all these results, it is clear that under the spacetime oscillations the field $\Phi$ itself starts performing oscillations in space and time with a certain momentum and frequency by following the resonance condition for the given $\omega$, where the $\phi_{1}$ field oscillates with frequency $\omega$ and $\phi_{2}$ oscillates with frequency $\omega / 2$. This shows that the field $\Phi$ undergoes parametric resonance under the spacetime oscillations.

\section{B. Frequency dependence of generation of field excitations}

As discussed earlier, to generate longitudinal excitation dominantly, the frequency of spacetime oscillations should be $\omega>m_{\Phi}$. Contrary to this, to generate transverse excitation of the field, there is no such frequency cutoff as the mass of modes corresponding to this excitation is zero. Therefore, even in the low-frequency regime, i.e., $\omega<m_{\Phi}$, spacetime oscillations can lead to the generation of transverse excitation via parametric resonance. Thus, there is a fundamental difference in the evolution of field in the frequency regimes $\omega>m_{\Phi}$ and $\omega \leq m_{\Phi}$, where in both the regimes, in principle, the field excitations can be generated under the spacetime oscillations.
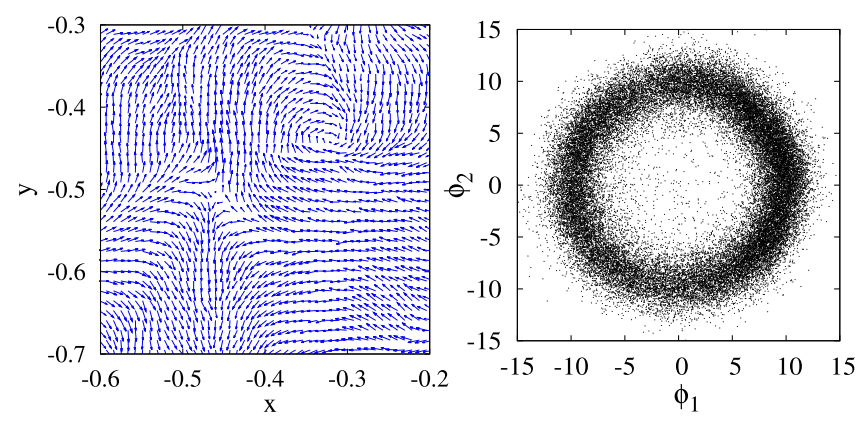

FIG. 10. The figure shows the field configuration in physical space (left panel) and in field space (right panel) at time $t=$ $18.5 \Lambda$ for the frequency $\omega=20 \Lambda^{-1}\left(\varepsilon=0.4, \Phi_{0}=10 \Lambda^{-1}\right.$, and $\lambda=40$ ). The left plot shows the formation of well-separated vortex-antivortex pair in the system. The right plot shows that at this frequency of spacetime oscillations mainly transverse excitation of the field has been generated.

In our simulation, we have studied the generation of field excitations and the formation of vortex-antivortex pairs for a wide range of frequencies of spacetime oscillations. At low frequencies, vortex-antivortex pairs form with relatively smaller vortex densities and remain well separated. In this frequency regime, unlike the case of high frequency, the field remains close to the VEV of the effective potential for significantly longer time of field evolution. This is shown in Fig. 10, where the left panel shows the vector plot of field configuration in the physical space and the right panel shows the field distribution in the field space; both are at time $t=18.5 \Lambda$. For this simulation, the spacetime oscillation frequency is taken as $\omega=20 \Lambda^{-1}$, and other parameters of the simulation are $\varepsilon=0.4, \Phi_{0}=10 \Lambda^{-1}$, and $\lambda=40$ (the same as used earlier). The left plot clearly shows the formation of the well-separated vortex-antivortex pair in the system. The right plot shows that at almost all lattice points the field is close to the VEV of the effective potential, where the field distribution covers the whole vacuum manifold at this time [at initial time, the field distribution is a localized distribution in the field space; see Fig. 2(a)]. It shows that mainly transverse excitation has been generated in the system, while longitudinal excitation is strongly suppressed in this frequency regime; compare with Fig. 2(d).

In the extremely-low-frequency regime of spacetime oscillations $\left[\omega \lesssim 4 \pi(\text { system size })^{-1}\right.$; see the discussion below], the finite size of the system affects the generation of field excitations. This happens because spacetime oscillations couple to $k$ modes of the field, where for the $\phi_{2}$ field $\left(k_{x(y)}=\omega / 2, k_{y(x)}=0\right)$ modes dominantly grow with time; see Figs. 4 and 5. For a finite lattice, the $k$ mode with a wavelength equal to the system size $L$ will be $k_{L}=2 \pi / L$. Therefore, any frequency $\omega$ below the cutoff $2 k_{L}$ cannot lead to the growth of the most dominant resonance modes of the field; excitations with wavelength larger than the lattice size cannot grow resonantly. 
Therefore, to excite the field, the frequency of spacetime oscillations must be $\omega \geq 4 \pi / L$ or equivalently $f_{a} \geq 2 / L$, where $f_{a}=\omega / 2 \pi$. In our simulations, even with frequencies two or three times larger than this cutoff, the field finds difficulties in achieving excitations. We have checked that the field excitations, which cannot be generated on a small lattice with a given spacetime oscillation frequency, can be generated using a larger lattice with the same frequency. This suggests that, in principle, any low frequency of spacetime oscillations can excite the field, leading to the formation of vortices if a suitably large system size is chosen and the field is evolved for a significantly long time; see details in the next subsection.

\section{Time dependence of formation of vortices on parameters of spacetime oscillations}

In this subsection, we present our study on the time dependence of formation of vortices on parameters of spacetime oscillations. For this, we note down time at which first vortex-antivortex pair forms in the system. We call this time as the vortex formation time and denote it by

$t_{\text {vortex }}$. To locate the vortices, we compute the variation of phase of $\Phi$ around each elementary square of the lattice. The variation of phase between any two adjacent points on the elementary square is determined by the geodesic rule [26]. By continuity, the phase of a complex scalar field along a loop is always $2 \pi n$, where $n$ is an integer. Throughout the evolution of the field, we observe only $n=0, \pm 1$ for the variation of phase around each elementary square, where we identify vortex with $n=+1$ and antivortex with $n=-1$. In our simulations, we observe that $t_{\text {vortex }}$ does not depend much on the amplitude of fluctuations (value of $\beta$ ) of the initial field configuration.

In Fig. 11, we plot $t_{\text {vortex }} \omega / 2 \pi$ versus $\omega$ for $\varepsilon=0.4$. The parameters of the effective potential are $\Phi_{0}=10 \Lambda^{-1}$ and $\lambda=40$. Note that $t_{\text {vortex }} \omega / 2 \pi$ is a dimensionless number which is equal to the number of cycle of spacetime oscillations up to the time $t_{\text {vortex }}$. The figure clearly shows that in the frequency regime $\omega \gtrsim 50 \Lambda^{-1}$ the number of cycle required to form vortices is almost independent from $\omega$. However, by decreasing the frequency of spacetime oscillations below $\omega=50 \Lambda^{-1}$, this number starts increasing. We have not seen the formation of any vortices below $\omega=16 \Lambda^{-1}$ up to a significantly long time of simulation on the used lattice.

As mentioned earlier, in extremely-low-frequency regime, the finite size of the system restricts the generation of field excitations; therefore, it must also restrict the formation of vortices. In our simulation, we observe that up to a significantly long time there is no formation of any vortex-antivortex pair on the $200 \times 200$ lattice with frequency $\omega=15 \Lambda^{-1}$; however, with the same parameters of simulation, vortices form on the $800 \times 800$ lattice. Therefore, it is quite possible that in Fig. 11 the deviations of $t_{\text {vortex }} \omega / 2 \pi$ below $\omega=50 \Lambda^{-1}$ from a constant value

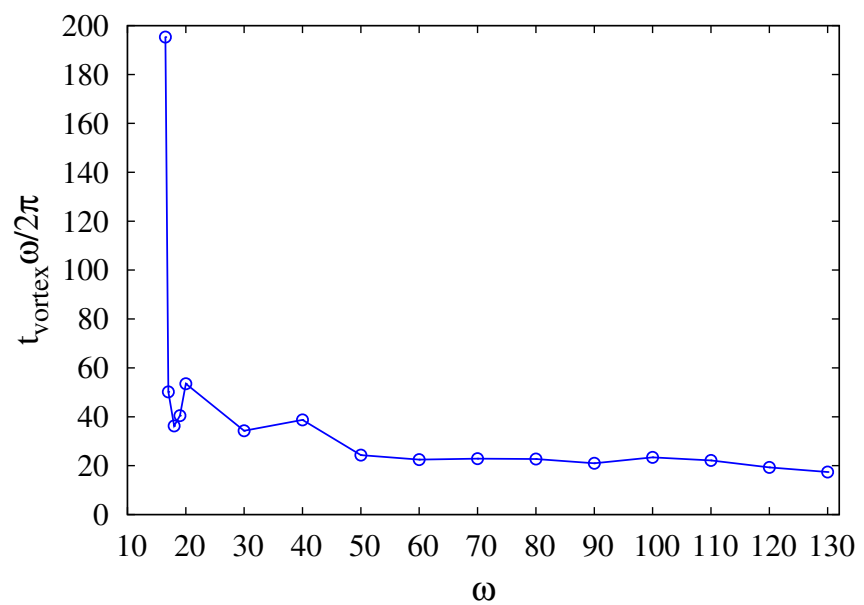

FIG. 11. The figure shows the dependence of vortex formation time $t_{\text {vortex }}$ on various frequencies $\omega$. In the figure, we plot the number of cycle of spacetime oscillations up to the time $t_{\mathrm{vortex}}$, i.e., $t_{\text {vortex }} \omega / 2 \pi$, vs $\omega$. Other parameters of simulation are $\varepsilon=0.4$, $\Phi_{0}=10 \Lambda^{-1}$, and $\lambda=40$. The figure clearly shows that in the frequency regime $\omega \gtrsim 50 \Lambda^{-1}, t_{\text {vortex }} \omega / 2 \pi$ is almost independent from $\omega$. However, by decreasing frequency below $\omega=50 \Lambda^{-1}$, this number starts increasing.

have arisen due to the finite size effects of the system. Therefore, it may also be possible that this constant value of $t_{\text {vortex }} \omega / 2 \pi$ is a universal number of cycle for formation of vortices and has the same value even in the low-frequency regime when finite size effects are eliminated.

To investigate this in more detail, we have performed simulations at different system sizes $L$, frequencies $\omega$, and boundary conditions. As mentioned earlier, in our simulations, we use periodic boundary conditions (PBCs). These boundary conditions affect our simulation results very strongly in the regime where finite size effects dominate. In general, we observe finite size effects when the frequency of spacetime oscillations becomes $\omega \lesssim 4 \pi / L$ or equivalently when $L \omega / 4 \pi \lesssim 1$. To show these effects explicitly and effects of boundary conditions in general, we study the effects of changing lattice size $L$ and choice of boundary condition on $t_{\text {vortex }}$ by keeping $\omega$ and $\varepsilon$ fix. We show below that values of $t_{\text {vortex }}$ using fixed boundary conditions (FBCs) are relatively less affected with finite size effects in comparison with simulations using PBCs. In Fig. 12, we plot $t_{\text {vortex }} \omega / 2 \pi$ versus $L \omega / 4 \pi$ (both are dimensionless numbers) for two different boundary conditions and at two frequencies $\omega, \omega_{1}=100 \Lambda^{-1}$ and $\omega_{2}=50 \Lambda^{-1}$, where for each curve $\omega$ and $\varepsilon(=0.4)$ are fixed. The parameters of effective potential are $\Phi_{0}=$ $10 \Lambda^{-1}$ and $\lambda=40$. In this figure, blue (circle) and black (square) lines correspond to simulations using PBCs at frequencies $\omega_{1}$ and $\omega_{2}$, respectively, while red (triangle) and brown (star) lines correspond to simulations using FBCs, again, at frequencies $\omega_{1}$ and $\omega_{2}$, respectively. Under the spacetime oscillations, the lowest cutoff for $L \omega / 4 \pi$ to 


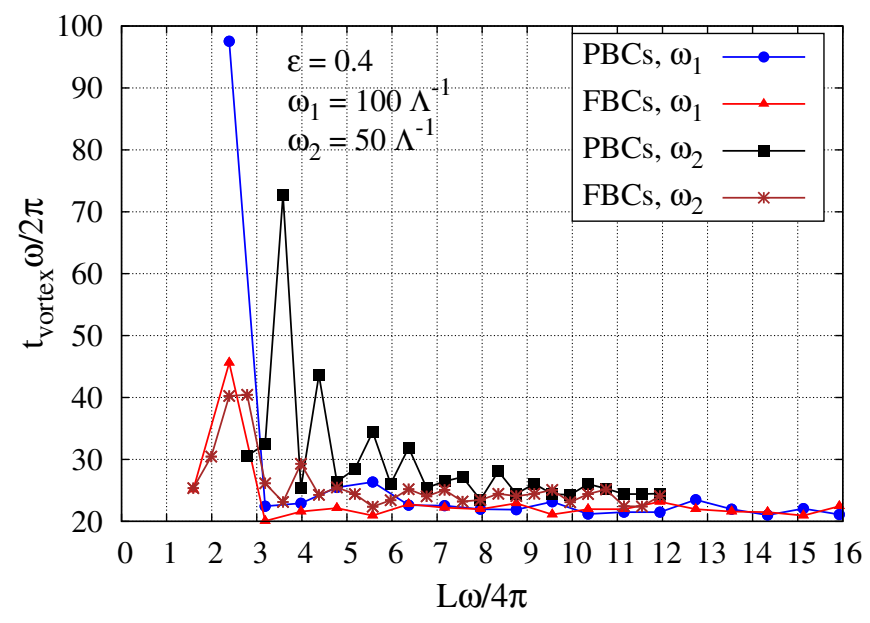

FIG. 12. The figure shows the effects of using different boundary conditions on $t_{\text {vortex }}$ at different system sizes $L$ and at two different $\omega, \omega_{1}=100 \Lambda^{-1}$ and $\omega_{2}=50 \Lambda^{-1}$, where for each curve $\omega$ and $\varepsilon$ are fixed. The parameters of the effective potential are $\Phi_{0}=10 \Lambda^{-1}$ and $\lambda=40$. Blue (circle) and black (square) lines correspond to simulations using periodic boundary conditions at frequencies $\omega_{1}$ and $\omega_{2}$, respectively, while red (triangle) and brown (star) lines correspond to simulations using fixed boundary conditions, again, at frequencies $\omega_{1}$ and $\omega_{2}$, respectively. In each curve, beyond the lowest value of $L \omega / 4 \pi$, $t_{\text {vortex }}$ diverges.

generate field excitations is 1 , which must also be a cutoff for formation of vortices in the system. Therefore, when $L \omega / 4 \pi$ approaches 1 from higher values, one would expect a growth in $t_{\mathrm{vortex}} \omega / 2 \pi$, which must diverge at $L \omega / 4 \pi \lesssim 1$. This is what we see in the figure, where in each curve, beyond the lowest value of $L \omega / 4 \pi, t_{\text {vortex }}$ diverges.

In the figure, for sufficiently large values of $L \omega / 4 \pi$ (say, greater than approximately 10$), t_{\mathrm{vortex}} \omega / 2 \pi$ is almost invariant with increasing value of $L \omega / 4 \pi$ and takes a constant value $\simeq 23$. This constant value seems to be a universal number, which is independent from choice of boundary condition and $\omega$. On the other hand, when $L \omega / 4 \pi$ approaches 1 (say, becomes less than approximately 10), $t_{\text {vortex }} \omega / 2 \pi$ starts increasing due to finite size effects and diverges when $L \omega / 4 \pi$ reaches very close to 1 (say, $\simeq 2$ for $\mathrm{PBCs}$ and $\simeq 1.5$ for FBCs). Note that in the case of FBCs the deviation in $t_{\mathrm{vortex}} \omega / 2 \pi$ from such a universal value is less in comparison with PBCs. Also, there are small lattice sizes, for which vortices are not formed using PBCs, whereas they are formed using FBCs. Thus, with FBCs, vortices could be formed easily in comparison with PBCs. It should be appreciated that for both boundary conditions, the number of cycles of spacetime oscillations required to form the first vortex-antivortex pair in the system is approximately the same at $L \omega / 4 \pi \gg 1$ and is independent of frequency $\omega$. Thus, this study clearly indicates that there is a universal number of cycle of spacetime oscillations to form the first vortex-antivortex pair in the system if there are no finite size effects. All in

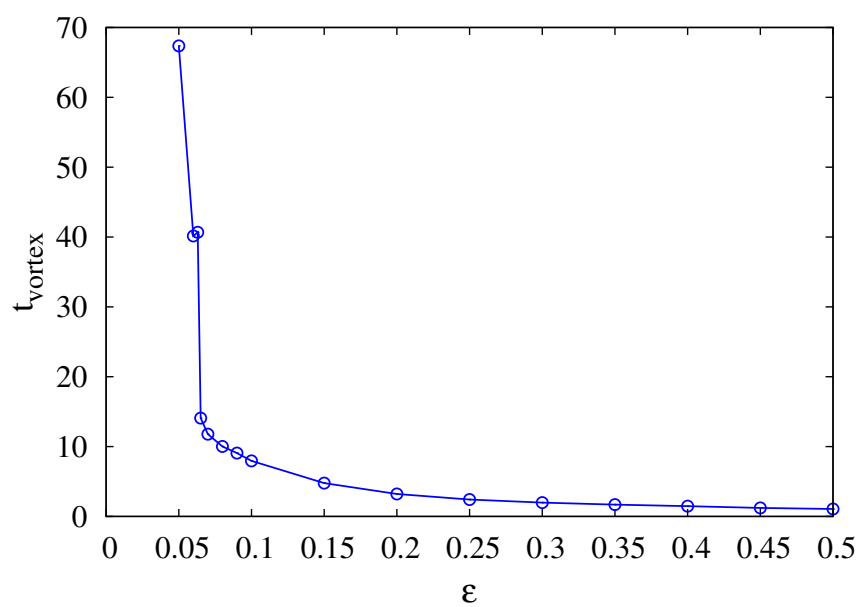

FIG. 13. The figure shows the effect of $\varepsilon$ on $t_{\text {vortex }}$. Other parameters of simulations are $\omega=100 \Lambda^{-1}, \Phi_{0}=10 \Lambda^{-1}$, and $\lambda=40$.

all, this investigation suggests that, even with a small frequency $\omega$, the field may get excited (transverse excitation) under the spacetime oscillations if the simulation is performed on a sufficiently large lattice $(L \gg 4 \pi / \omega)$ and the field is evolved for a significantly long time $\left(t \gtrsim t_{\text {vortex }} \simeq 46 \pi / \omega \simeq \frac{3}{2} \frac{\pi^{4}}{\omega}\right)$.

In Fig. 13, we plot $t_{\text {vortex }}$ versus $\varepsilon$. Other parameters of simulations are $\omega=100 \Lambda^{-1}, \Phi_{0}=10 \Lambda^{-1}$, and $\lambda=40$. The figure clearly shows that decreasing $\varepsilon$ causes more time to form a vortex-antivortex pair in the system. For lower values of $\varepsilon$, the system takes a longer time to achieve field excitations, where the large amplitude field oscillation stage persists for a longer time. In the regime of very small $\varepsilon$, the field excitations do not grow sufficiently to create vortices in the system. This behavior of the field response to $\varepsilon$ is also a feature of parametric resonance, in which the resonance growth is strongly suppressed by decreasing the amplitude of time-dependent parameter(s) of the oscillator [25].

\section{Formation of vortex-antivortex lattice under spacetime oscillations}

The formation of vortex-antivortex lattice structure has interest in various condensed matter systems. In Refs. [27-29], the formation of such lattices is studied in (i) superconducting films with magnetic pinning arrays, (ii) ultracold fermionic gases in two dimensions, and (iii) superconducting twisted-bilayer graphene. Melting of the vortex-antivortex lattice in two-dimensional Fermi gases has been studied in Ref. [30]. For certain parameters of the simulation, we have also seen the formation of vortex-antivortex lattice structures under spacetime oscillations. In Fig. 14, we show the phase (left panel) and magnitude (right panel) of field $\Phi$ at two different times of field evolution. The parameters of the simulation 

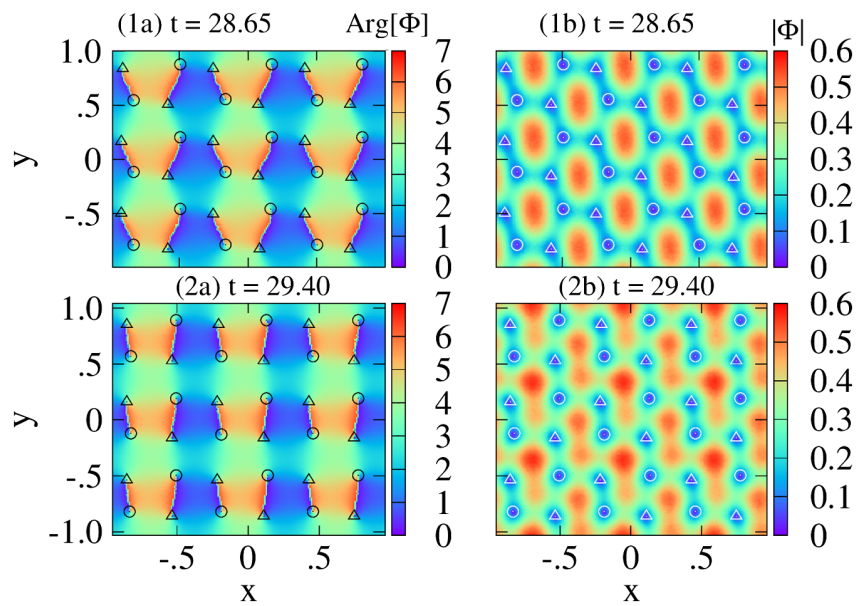

FIG. 14. The figure shows the formation of vortex-antivortex lattice structures in the system. The locations of the vortex and antivortex in the plots are depicted by circles and triangles, respectively. The parameters of the simulation are $\omega=17 \Lambda^{-1}$, $\varepsilon=0.4, \Phi_{0}=0.1 \Lambda^{-1}$, and $\lambda=4$. Plots $1 \mathrm{a}$ and $1 \mathrm{~b}$ show the phase and magnitude of field $\Phi$ at time $t=28.65 \Lambda$, while plots $2 \mathrm{a}$ and $2 \mathrm{~b}$ show the same at time $t=29.4 \Lambda$.

are $\omega=17 \Lambda^{-1}, \varepsilon=0.4, \Phi_{0}=0.1 \Lambda^{-1}$, and $\lambda=4$. As mentioned earlier, for $\omega \gg m_{\Phi}$, the formed vortices under spacetime oscillations are not topological in nature. For the above parameters of the simulation also, $\omega$ is much greater than $m_{\Phi}$; therefore, the formed vortices in Fig. 14 do not satisfy the properties of topological vortices. The core size of these vortices is much less than $m_{\Phi}^{-1}$; rather, it is given by approximately $\omega^{-1}$, where the field values outside these vortices are six times greater than the VEV. Figures 14(1a) and 14(1b) show the phase and magnitude of the field $\Phi$ at time $t=28.65 \Lambda$, while Figs. 14(2a) and 14(2b) show the same at time $t=29.4 \Lambda$. On the plots, circles and triangles indicate the locations of vortices and antivortices, respectively. In the left panel, these locations correspond to $+2 \pi$ and $-2 \pi$ variations of phase of $\Phi$, respectively, and in the right panel, they correspond to the places where the magnitude of $\Phi$ is zero. One can clearly see that there are vortex-antivortex lattice structures in both, upper and lower, panels. We observe that these lattice structures keep changing with time. Formation of these structures does not depend upon the amplitude of initial fluctuations, i.e., on the value of $\beta$. It may be possible that such lattice structures are arising because of the use of periodic boundary conditions in the simulation.

\section{CONCLUSIONS}

In this work, we have performed $(2+1)$-dimensional simulations for a complex scalar field in the presence of oscillating spacetime metric background. We have considered the spacetime metric as periodic time-dependent perturbations on the top of the Minkowski metric. The field is taken on the VEV of the U(1) symmetry broken effective potential, where small random fluctuations in the initial field configuration have been considered. We find that, depending upon the amplitude and frequency of metric oscillations, the field undergoes parametric resonance, which leads to the generation of field excitations and the formation of vortex-antivortex pairs in the system. There is a fundamental difference in the evolution of field in the frequency regimes $\omega>m_{\Phi}$ and $\omega \leq m_{\Phi}$. In the lowfrequency regime, mainly transverse excitation arises, and well-separated vortex-antivortex pairs form in the system, whereas in the high-frequency regime, longitudinal excitation of the field also arises prominently.

In our simulation, finite size effects restrict the generation of field excitations in the regime $L \omega / 4 \pi \lesssim 1$, where $L$ is the system size and $\omega$ is the frequency of spacetime oscillations. Our study suggests that, in principle, any low frequency can excite the field if a suitable system size is chosen $(L \gg 4 \pi / \omega)$ and the field is evolved for a significantly long time $\left(t \gtrsim \frac{3}{2} \frac{\pi^{4}}{\omega}\right)$. We have studied the effects of frequency, boundary conditions, and system size on the number of cycle of spacetime oscillations required to form the first vortex-antivortex pair in the system. This suggests that there is a universal number of cycle of spacetime oscillations for formation of the first vortex-antivortex pair in the system if there are no finite size effects. The formation of vortices also depends upon the amplitude of spacetime oscillations; the vortex formation time increases by decreasing amplitude. For certain parameters of the simulation, we have seen the formation of vortexantivortex lattice structures. However, such vortices do not satisfy the properties of topological vortices as in such case $\omega$ is much greater than $m_{\Phi}$ (mass of the field), for which the generated field modes have much larger momentum in comparison with $m_{\Phi}$. Only in the case of $\omega \lesssim m_{\Phi}$, the formed vortices under spacetime oscillations are topological in nature.

In this work, we have ignored an important aspect of the system evolution, which is the backreaction of energy density variations of field on the spacetime metric. As we have shown, under spacetime oscillations, vortices are formed, which have an energy density profile, with a maximum value in the vortex core. Therefore, depending upon the energy of vortex configuration, the spacetime manifold itself may be affected by these energy density variations, which may affect the further evolution of the field. It would be interesting to see the field evolution under such a complete scenario. We will try to pursue this in future.

To study the time evolution of condensate field of neutron stars superfluidity during BNS merger, a full $(3+1)$-dimensional simulation with an appropriate timedependent deformation of the star metric is required. It will reveal whether the timescale and length scale of the whole process are sufficient to excite the condensate field to lead the formation of vortex-antivortex pairs in the interior superfluid. Our present study suggests that there is no 
possibility of the generation of condensate excitations of neutron star superfluidity under the phenomenon of parametric resonance due to finite size effects. However, a detailed investigation is needed to reveal whether there is any other method of generation of such excitations.

The present work has applications in other systems also. The second homotopy group of the $S^{2}$ manifold is nontrivial, i.e., $\pi_{2}\left(S^{2}\right)=\mathcal{Z}$ [3], which allows the existence of topological texture in $(2+1)$ dimensions (baby Skyrmion) and topological monopole in $(3+1)$ dimensions. Similarly, the $S^{3}$ manifold [group space of $S U(2)$ ] allows the existence of topological texture in $(3+1)$ dimensions as the third homotopy group is nontrivial, i.e., $\pi_{3}\left(S^{3}\right)=\mathcal{Z}$ [3]. These topological objects have cosmological interests, the formation of which could be studied under spacetime oscillations as well (similar to the formation of vortices studied in this paper). In the Abelian-Higgs model and pure $U(1)$ gauge theory also, one could study the formation of flux tubes and generation of magnetic field, respectively, under these oscillations.

This study can also be extended to the U(1) symmetry broken effective potential with a small explicit symmetry breaking term. In such a case, there must be a lowest-frequency cutoff of spacetime oscillations for the generation of field excitations set by the mass of pseudoGoldstone modes; only frequencies higher than or equal to the mass of these modes can lead to the field excitations. In our simulation, we have seen the generation of field excitations in the presence of such a term (we have not presented this here). This result suggests a possibility of generation of excitations in the axionlike field having mass approximately $10^{-22}-10^{-21} \mathrm{eV}$ [31] and coupled with spacetime oscillations. The axionlike particles having the above mass range are considered to be a possible candidate of dark matter, known as the "wave dark matter" or "fuzzy dark matter" [31]. To excite this field, correspondingly, the frequency of spacetime oscillations must be greater than or approximately equal to $0.1-1 \mu \mathrm{Hz}$. This frequency can be easily achieved by BNS [14] and black hole merger [32] systems, which produce gravitational waves in the frequency range of approximately $10 \mathrm{~Hz}-1 \mathrm{kHz}$ with a significantly large strain amplitude. We expect that sustained spacetime oscillations will give rise excitation in axionlike field over a length scale having momentum of field corresponding to the frequency of spacetime oscillations. The effect will be more pronounced in the nearest region of gravitational wave source as the strain amplitude is significantly large there. If such an excitation arises, then as soon as gravitational waves pass completely, the field will start rolling toward the minima of effective potential and perform coherent oscillations at various constant $r$ hypersurfaces about the source. We will present this phenomenon in detail in our future work.

\section{ACKNOWLEDGMENTS}

We are very grateful to Ajit M. Srivastava and Subhroneel Chakrabarti for very useful discussions, suggestions, and comments on the manuscript. We also would like to thank A. P. Balachandran, P. S. Saumia, Abhishek Atreya, T. P. Sreeraj, Nirupam Dutta, and Minati Biswal for useful discussions and comments on the work.
[1] W. H. Zurek, Phys. Rep. 276, 177 (1996).

[2] A. Rajantie, Int. J. Mod. Phys. A 17, 1 (2002).

[3] N. D. Mermin, Rev. Mod. Phys. 51, 591 (1979).

[4] T. W. B. Kibble, J. Phys. A 9, 1387 (1976); Phys. Rep. 67, 183 (1980).

[5] V. S. Kumar, B. Layek, A. M. Srivastava, S. Sanyal, and V. K. Tiwari, Int. J. Mod. Phys. A 21, 1199 (2006).

[6] S. S. Dave and A. M. Srivastava, Europhys. Lett. 126, 31001 (2019).

[7] L. D. Landau and E. F. Lifshitz, Statistical Physics Part 2 (Pergamon, New York, 1980).

[8] D. R. Tilley and J. Tilley, Superfluidity and Superconductivity, 3rd ed. (Graduate Student Series in Physics, Institute of Physics Publishing, Bristol and Philadelphia, 2005).

[9] S. Digal, R. Ray, S. Sengupta, and A. M. Srivastava, Phys. Rev. Lett. 84, 826 (2000).

[10] R. Ray and S. Sengupta, Phys. Rev. D 65, 063521 (2002).
[11] E. A. L. Henn, J. A. Seman, G. Roati, and K. M. F. Magalhães, and V.S. Bagnato, Phys. Rev. Lett. 103, 045301 (2009); E. A. L. Henn, J. A. Seman, E. R. F. Ramos, M. Caracanhas, P. Castilho, E. P. Olímpio, G. Roati, D. V. Magalhães, K. M. F. Magalhães, and V. S. Bagnato, Phys. Rev. A 79, 043618 (2009).

[12] V. I. Yukalov, A. N. Novikov, and V. S. Bagnato, Phys. Lett. A 379, 1366 (2015).

[13] V. I. Yukalov, E. P. Yukalova, and V. S. Bagnato, Phys. Rev. A 66, 043602 (2002).

[14] B. P. Abbott et al. (LIGO Scientific Collaboration and Virgo Collaboration), Phys. Rev. Lett. 119, 161101 (2017).

[15] R. E. Packard, Phys. Rev. Lett. 28, 1080 (1972).

[16] D. G. Yakovlev, A. D. Kaminker, and K. P. Levenfish, Astron. Astrophys. 343, 650 (1999), http://cdsarc.ustrasbg.fr/ftp/vizier/aa/papers/9343002/2300650.pdf.

[17] M. G. Alford, A. Schmitt, K. Rajagopal, and T. Schäfer, Rev. Mod. Phys. 80, 1455 (2008). 
[18] C. N. Colacino, Gribov-80 Memorial Volume; Quantum Chromodynamics And Beyond (World Scientific, Singapore, 2010), pp. 449-454.

[19] A. Perego, S. Bernuzzi, and D. Radice, Eur. Phys. J. A 55, 124 (2019).

[20] L. Amundsen and E. Østgaard, Nucl. Phys. A437, 487 (1985); A442, 163 (1985).

[21] J. Anandan, Phys. Rev. Lett. 47, 463 (1981).

[22] A. Schmitt, Introduction to Superfluidity; Field-theoretical Approach and Applications (Springer, New York, 2015).

[23] C. Xiong, M. R. R. Good, Y. Guo, X. Liu, and K. Huang, Phys. Rev. D 90, 125019 (2014).

[24] S. Carroll, Spacetime and Geometry; An Introduction to General Relativity (Addison-Wesley, Reading, MA, 2004).
[25] L. D. Landau and E.F. Lifshitz, Mechanics, 3rd ed. (Elsevier, New York, 2007).

[26] M. J. Bowick, L. Chandar, E. A. Schiff, and A. M. Srivastava, Science 263, 943 (1994).

[27] M. V. Milošević and F. M. Peeters, J. Low Temp. Phys. 139, 257 (2005).

[28] S. S. Botelho and C. A. R. Sá de Melo, Phys. Rev. Lett. 96, 040404 (2006).

[29] Y. Su and S.-Z. Lin, Phys. Rev. B 98, 195101 (2018).

[30] G. Cao, L. He, and X.-G. Huang, Phys. Rev. A 96, 063618 (2017).

[31] L. Hui, J. P. Ostriker, S. Tremaine, and E. Witten, Phys. Rev. D 95, 043541 (2017).

[32] B. P. Abbott et al. (LIGO Scientific Collaboration and Virgo Collaboration), Phys. Rev. Lett. 116, 061102 (2016). 